\title{
Laser control for the optimal evolution of pure quantum states
}

\author{
D. Sugny ${ }^{1}$ * A. Keller ${ }^{2}$, O. Atabek ${ }^{2}$, D. Daems ${ }^{3}$, C. M. Dion ${ }^{4}$, S. Guérin ${ }^{1}$, and H. R. Jauslin ${ }^{1}$ \\ 1 Laboratoire de Physique de l'Université de Bourgogne, \\ UMR CNRS 5027, BP 47870, 21078 Dijon, France \\ 2 Laboratoire de Photophysique Moléculaire du CNRS, Université Paris-Sud, \\ Bât. 210 - Campus d'Orsay, 91405 Orsay Cedex, France \\ 3 Center for Nonlinear Phenomena and Complex Systems, \\ Université Libre de Bruxelles, 1050 Brussels, Belgium and \\ 4 Department of Physics, Umeå University, SE-90187 Umeå, Sweden
}

\begin{abstract}
Starting from an initial pure quantum state, we present a strategy for reaching a target state corresponding to the extremum (maximum or minimum) of a given observable. We show that a sequence of pulses of moderate intensity, applied at times when the average of the observable reaches its local or global extremum, constitutes a strategy transferable to different control issues. Among them, post-pulse molecular alignment and orientation are presented as examples. The robustness of such strategies with respect to experimentally relevant parameters is also examined.
\end{abstract}

PACS numbers: $33.80 .-\mathrm{b}, 32.80 . \mathrm{Lg}, 42.50 . \mathrm{Hz}$

\section{INTRODUCTION}

Recent advances in laser technology have opened up large possibilities for the control of quantum processes playing a role in a variety of problems encompassing chemical reactivity 1, 2], high-order harmonic generation [3], or logical gates with applications in quantum information [4, 5, [6]. Several advances have been achieved in this context, from the discovery of elementary basic mechanisms of strong field induced molecular dynamics, to optimal control strategies bringing together sophisticated numerical algorithms (such as evolutionary algorithms) [3, 7]. Parallel to these works, a systematic study of quantum systems in either pure or mixed (statistical) states has been undertaken using control theory [8]. Upper and lower bounds corresponding to kinematical constraints have been established for the expectation value of arbitrary observables of driven quantum systems [9, 10], with a particular attention paid to their dynamical realizability [11, 12]. This latter question, which is related to their complete controllability, has been solved for linear control, i.e., for Hamiltonians depending in a linear way on the control functions, of non-dissipative finite-level quantum systems [1]].

The aim of this study, which is a continuation of our previous paper [13], is to show how a strategy worked out for the laser control of a specific observable can be transposed to a generic system. An essential feature of the control scheme we propose is to reduce the physical Hilbert space to a subspace $\mathcal{H}^{(N)}$, of finite dimension $N$. A target state $\left|\chi^{(N)}\right\rangle$, which maximizes (or minimizes) the expectation value of an observable $\mathcal{O}$, is then defined in this subspace. The first obvious advantage of the finite dimensionality being that $\left|\chi^{(N)}\right\rangle$ appears, through

*Electronic address: dominique.sugny@u-bourgogne.fr a variational principle, to be the eigenvector (for the non-degenerate case) associated to $\mathcal{H}^{(N)}$ with the highest or lowest eigenvalue of the restriction of the observable $\mathcal{O}$. These bounds being kinematic, they only indicate to which extent the system can be dynamically controlled by application of time-dependent external fields. Moreover, in the finite-dimensional case, the complete or noncomplete controllability can be determined and related (for a linear control system) to the dimension of the Lie algebra generated by the unperturbed Hamiltonian and the interaction operator [11, 12.

Assuming the dynamical realizability of the target state, the remaining question consists in explicitly finding a suitable scheme in terms of a unitary evolution operator $U(t)$ which, starting from an initial pure state of the molecular system, dynamically brings it close to the state $\left|\chi^{(N)}\right\rangle$. Several control strategies have already been proposed using, for instance, adiabatic passage techniques 14, 15], factorizations of unitary operators [16] or optimal control schemes [7, 17]. Here, we pursue another approach and we show that a sequence of short pulses of moderate intensity can be devised for reaching these target states. A generalization to models incorporating fluctuating environmental effects such as temperature, via statistically mixed initial states, is still an open question on which work is in progress.

The strategy is exemplified by two illustrative cases, the alignment and the orientation control of rigid-rotor molecules. We show that either complete or close to complete control is achieved after the application of a finite number of pulses. More precisely, the alignment / orientation control processes, aiming both at efficiency and maximal duration within a rotational period, require few kicks to get close to the target, while remaining in the conventionally fixed finite dimensional Hilbert subspace. We note that in all the dynamical mechanisms under consideration, we are interested in controlling the post-pulse behavior of the observables. The motivations for such 
processes are now well established in a wide variety of applications extending from chemical reaction dynamics to surface processing, catalysis, nanoscale design, and quantum computing [2, 18, 19, 20, 21].

The paper is organized as follows. A mathematical setup is given in Sec. II for the general control strategy, which is then applied to the case of molecular alignment / orientation. These processes are taken on a parallel footing in Sec. III] by considering either the polarizability or the permanent dipole moment of the molecular system. Section IV collects the results with a special emphasis on the robustness with respect to control parameters and the experimental feasibility. Concluding remarks and prospective views are presented in Sec. $\nabla$ Some details of the calculations and some proofs are presented in Appendices $\mathrm{A}, \mathrm{B}, \mathrm{C}$ and D

\section{METHODOLOGY}

The field-free Hamiltonian acting on the physical Hilbert space $\mathcal{H}$ is denoted $H_{0}$. The initial pure quantum state of interest $\left|\psi_{n}(t=0)\right\rangle$, is taken as one of the eigenstates of $H_{0}$ corresponding to the eigenvalue $E_{n}$. One considers an observable $\mathcal{O}$ which, through the dynamical behavior of its expectation value $\langle\mathcal{O}(t)\rangle$, describes the physical property to be controlled by the external field. $\mathcal{O}$ is a self-adjoint operator which does not commute with $H_{0}$. The aim of the control is to find an evolution operator $U(t)$, within a class of experimentally realizable processes, such that the time evolution of the initial state under the action of $U(t)$ leads to an average $\left\langle\psi_{n}(t)|\mathcal{O}| \psi_{n}(t)\right\rangle$ that is maximized or minimized. This goal is achieved in three steps.

\section{A. Finite dimensional subspace}

We first select a finite $N$-dimensional subspace $\mathcal{H}^{(N)}$ of the physical Hilbert space. For instance, in the case of alignment / orientation such a subspace is generated by the first $N$ eigenstates $|n\rangle$ of $H_{0}$ with eigenvalues $E_{n}$ [13]. The mathematical advantage of this reduction is twofold when considering the reduced operator

$$
\mathcal{O}^{(N)}=P^{(N)} \mathcal{O} P^{(N)},
$$

$P^{(N)}$ being the projector on the subspace $\mathcal{H}^{(N)}$. The first advantage, of kinematical nature [9, 10], is related to the fact that $\mathcal{O}^{(N)}$ has now an upper and lower bounded discrete spectrum, as opposed to the possibly continuous or unbounded spectrum of $\mathcal{O}$. The second is that the controllability of the system can be completely analyzed (see Sec.IID for details), whereas such results are limited for the infinite-dimensional case [22].

Finally, we assume that all the fundamental frequencies of $H_{0}$ in $\mathcal{H}^{(N)}$ are commensurate, i.e., integer multiples of a fundamental frequency $\omega$, which implies that the motion is periodic in time, with period $T=2 \pi / \omega$.
Apart from these mathematical considerations, which will be used in Sec. IID the reduction of the Hilbert space may also lead to other physical advantages in specific cases. Indeed, for post-pulse alignment / orientation control, the finite dimensionality of the molecular rotational space, involving low momenta, leads to longer alignment / orientation dynamics as a result of the slower oscillation of $\langle\mathcal{O}(t)\rangle$ after interaction with the field. It is precisely this behavior that roughly constitutes the basis of the compromise between efficiency and long duration of such control issues. Reciprocally, the justification of the dimensionality reduction is related to the fact that moderate perturbations, i.e., a moderate number of applied pulses and amplitude, can only transfer finite amounts of energy to the molecule, confining the system to a finite-dimensional subspace [13].

\section{B. Controllability}

The control is exerted through the application of a time-dependent external field described by the Hamiltonian

$$
H(t)=H_{0}+V(t),
$$

where $V(t)$ is the molecule-field interaction potential. The general structure of $V(t)=V(\vec{\xi}, \overrightarrow{\mathcal{E}}(t))$ involves the field vector $\overrightarrow{\mathcal{E}}(t)$ (amplitude and polarization direction) together with molecular vectorial characteristics $\vec{\xi}$ that may, in some cases, be field-induced (an example of such a behavior, through the polarizability interaction, is given in Sec. (III). Moreover, in order to apply the results of control theory, we also assume that the system is controllinear, which means here that $V$ can be written in the form

$$
V(t)=-v(t) H_{I}
$$

where $v$ is a real control function (that can be chosen at will) and $H_{I}$ the interaction operator. If a system of the form (3) is completely controllable [11, 12] then there exists a function $v(t)$ such that the corresponding propagator $U(t)$ brings the initial state to the target state. A simple example of a non-controllable system is a decoupled one. In this case, the matrix representation of $H$ is block-diagonal and the Hilbert space can be decomposed as a direct sum of at least two orthogonal subspaces. However, for non-decoupled systems, the question of dynamical realizability is more difficult and an investigation of the structure of the Lie algebra is needed. A Lie algebra of matrices is a subspace of the vector space (over the field of reals) of $N \times N$ matrices (with complex entries) which is stable under the commutation operation [1]. Two cases of particular relevance here are the Lie algebra of $N \times N$ skew-Hermitian matrices denoted by $u(N)$ and whose dimension is $N^{2}$, and the Lie algebra $s u(N)$ of $N \times N$ zero-trace skew Hermitian matrices, whose dimension is $N^{2}-1$. We recall [11, 12] that a necessary 
and sufficient condition for the complete controllability of the Hamiltonian system defined by $H$ [Eq. (2)] is that the Lie algebra generated by $i H_{0}$ and $i H_{I}$ be $u(N)$. In the rest of this section, we will assume the dynamical realizability of the target states.

\section{Target state}

The state $\left|\chi^{(N)}\right\rangle$ which maximizes (or minimizes) $\mathcal{O}^{(N)}$ in the subspace $\mathcal{H}^{(N)}$ is nothing but the eigenstate of $\mathcal{O}^{(N)}$ corresponding to its upper (or lower) eigenvalue. This provides a clear description of the target state in terms of a linear combination of states $|n\rangle$ in direct relation with the observable to control. If $N$ is large enough, the upper (or lower) eigenvalue of $\mathcal{O}^{(N)}$ will be close to the corresponding bound of $\mathcal{O}$ (if this operator is bounded) and an efficient control could be achieved (assuming complete controllability). Once again, it is worth noting that large $N$ allows for higher values of $\left|\left\langle\mathcal{O}^{(N)}\right\rangle\right|$, but leads to higher energy transfer from the external field to the molecular system and consequently results into shorter durations as the post-pulse average $\langle\mathcal{O}(t)\rangle$ is done over a superposition involving many more eigenstates of $H_{0}$.

\section{Control strategy}

We shall now present in detail two control schemes. The first one, which was first proposed in 23] and further developed in 13], consists in applying pulses each time $\left\langle\mathcal{O}^{(N)}\right\rangle$ reaches its maximum value within a period $T$ of the field-free dynamics. An individual pulse perturbs the molecule by an effective evolution operator $U_{\tilde{A}}$, where $\tilde{A}$ is a real parameter related to the field amplitude: Eq. (7) gives, for instance, the expression of $\tilde{A}$ in the sudden approximation. The system remains in the subspace $\mathcal{H}_{\tilde{A}}^{(N)}$, up to negligible corrections, if the field amplitude $\tilde{A}$ is small enough. Between two successive pulses, the molecule evolves following its field-free motion, governed by $U_{H_{0}}(\Delta t)=\exp \left(-i H_{0} \Delta t\right)$. An important hypothesis of our strategy is that the system is to be perturbed according to a unitary operator $U_{\tilde{A}}$, which commutes with $\mathcal{O}^{(N)}$, such that its application does not alter $\left\langle\mathcal{O}^{(N)}\right\rangle=\left\langle U_{\tilde{A}}^{-1} \mathcal{O}^{(N)} U_{\tilde{A}}\right\rangle$. Furthermore, the optimal target state $\left|\chi^{(N)}\right\rangle$ is an eigenfunction of both $\mathcal{O}^{(N)}$ and $U_{\tilde{A}}$.

We now consider that pulses are applied at times $t_{i}$ when $\left\langle\mathcal{O}^{(N)}(t)\right\rangle$ reaches its global maximum $\mathcal{O}_{i}=$ $\left\langle\mathcal{O}^{(N)}\left(t_{i}\right)\right\rangle$. The sequence of $\mathcal{O}_{i}$ 's is increasing (possibly constant) but bounded and is therefore convergent. Due to the hypothesis on the fundamental frequencies (Sec. IIA) and the fact that $\left\langle\mathcal{O}^{(N)}\right\rangle=\left\langle U_{\tilde{A}}^{-1} \mathcal{O}^{(N)} U_{\tilde{A}}\right\rangle$, $\left\langle\mathcal{O}^{(N)}(t)\right\rangle$ is a periodic, continuous and differentiable (under free-evolution) function. It is then easy to verify that the limit of the preceding sequence is a fixed point
$\mathcal{O}_{i}=\mathcal{O}_{i+1}$, corresponding to a wave function $\left|\psi_{f}\right\rangle$ (or a family of wave functions) such that $\left\langle\psi_{f}\left|\mathcal{O}^{(N)}\right| \psi_{f}\right\rangle=$ $\left\langle\psi_{f}\left|U_{\tilde{A}}^{-1} \mathcal{O}^{(N)} U_{\tilde{A}}\right| \psi_{f}\right\rangle=\mathcal{O}_{i}$ is a global maximum within a period $T$ for any value of the parameter $\tilde{A}$.

The description of this control strategy raises several questions which are not completely resolved. The first one is the set of limits (corresponding to the fixed points) of the process. It can readily be shown that this set contains some of the eigenvectors of the operator $\mathcal{O}^{(N)}$, but we can look for the conditions on $H_{0}, U_{\tilde{A}}$ and $\mathcal{O}^{(N)}$ such that these wave functions be the unique fixed points of the strategy. Appendix $\mathrm{A}$ provides a sufficient condition on these operators. The second question deals with the determination of the domain of attraction of each fixed point and, more precisely, the domain of the target state. The domain of attraction of $\left|\psi_{f}\right\rangle$ can be defined as the set of initial states $|\psi\rangle$ such that, for some choice of the parameters $\tilde{A}_{i}$ (the pulses being labeled by $i$ ), the process, starting from $|\psi\rangle$ converges to $\left|\psi_{f}\right\rangle$. The last open question concerns the reduction to a finite dimensional space of the dynamics. In this paper, the fact that the dynamics resides within the subspace $\mathcal{H}^{(N)}$ is only numerically justified (see Sec. III for details). Work is in progress on these open questions.

The second control scheme that can be used is a strategy similar in its spirit to the first one. Up to now, we have searched for a sequence of perturbations that maximize the average value of the observable $\left\langle\mathcal{O}^{(N)}(t)\right\rangle$. Another relevant optimization would be to get closer to the target state $\left|\chi^{(N)}\right\rangle$. This can be done in practice by applying pulses each time the modulus of the projection on the target state, $\left|\left\langle\chi^{(N)} \mid \psi_{n}(t)\right\rangle\right|$, reaches a maximum. The relative merits of the two control schemes will be discussed in Sec. IV]

To conclude, we mention an alternative that can favor the convergence of the control scheme. The general strategy is based on global maxima of the field-free dynamics, but local maxima can also be used. This will be illustrated numerically for the control of molecular alignment in Sec. IV where it will be shown that a better convergence is obtained with local maxima.

\section{E. The sudden approximation}

Depending on the duration $\tau$ of the radiative interaction $V(t)$ as compared to molecular characteristic times $T$, we may be in the adiabatic $(\tau \gg T)$ or in the sudden $(\tau \ll T)$ limits. Long laser pulses have been shown to yield very efficient adiabatic molecular control [24, 25, 26, 27], but with the caveat of it disappearing after the pulse, as the molecule returns to its initial state when the turn off is adiabatic. As we are rather concerned with the post-pulse behavior of $\langle\mathcal{O}(t)\rangle$, we hereafter use short pulses, assuming the sudden limit. Following the derivation of the lowest order impulsive approximation given in Refs. [28, 29], the time-dependent 
Schrödinger equation, written in the interaction representation as

$$
i \hbar \frac{\partial}{\partial t} \tilde{\psi}_{\lambda}(t)=-\left[e^{i H_{0} t / \hbar} V(t) e^{-i H_{0} t / \hbar}\right] \tilde{\psi}_{\lambda}(t),
$$

with $\tilde{\psi}_{n}(t)=e^{i H_{0} t / \hbar} \psi_{n}(t)$, is integrated on the total pulse duration $\tau$ to yield

$\tilde{\psi}_{n}(\tau)=\frac{i}{\hbar} \int_{0}^{\tau}\left[e^{i H_{0} t / \hbar} V(t) e^{-i H_{0} t / \hbar}\right] \tilde{\psi}_{n}(t) d t+\tilde{\psi}_{n}(t=0)$.

At the lowest order in $\tau / T$, one can substitute $e^{i H_{0} t / \hbar}$ with 1 in Eq. (5) and, using $V(t)=-v(t) H_{I}$ [Eq. (3)], the integral equation (15) can be solved as

$$
\tilde{\psi}_{n}(\tau)=\exp \left(i \tilde{A} H_{I}\right) \tilde{\psi}_{n}(t=0),
$$

with

$$
\tilde{A}=\frac{1}{\hbar} \int_{0}^{\tau} v(t) d t
$$

Finally, returning to the Schrödinger representation, one obtains

$$
\psi_{n}(t \geq \tau)=U_{H_{0}}(t) U_{\tilde{A}} \psi_{n}(t=0),
$$

where the evolution operators are given by

$$
U_{H_{0}}(t)=\exp \left(-i H_{0} t / \hbar\right)
$$

and

$$
U_{\tilde{A}}=\exp \left(i \tilde{A} H_{I}\right) .
$$

We are now in a position to examine the conditions (choice of the parameters $N, A$, and $t_{i}$ 's) under which the alluded strategies work in the control of alignment / orientation processes.

\section{CONTROL OF THE MOLECULAR ALIGNMENT / ORIENTATION}

Laser-induced alignment and orientation are important issues in controlling molecular dynamics 2]. For a diatomic molecule driven by a linearly polarized laser field, alignment means an increased probability distribution along the polarization axis whereas orientation requires in addition the same (or opposite) direction as the polarization vector. In a variety of applications, extending from chemical reaction dynamics to surface processing, catalysis and nanoscale design [2, 18, 19, 21], noticeable orientation that persists after the end of the pulse is of special importance. This is why the identification of target states which fulfill these two requirements (i.e., efficiency and persistence) is the basis of the construction presented in Ref. [13].

The time evolution of the molecule, hereafter described in a 3D rigid rotor approximation, interacting with a linearly-polarized field is governed by the time-dependent Schrödinger equation (TDSE), expressed in atomic units,

$$
i \frac{\partial}{\partial t} \psi(\theta, \phi ; t)=\left[B J^{2}-\vec{\mu}(\overrightarrow{\mathcal{E}}) \cdot \overrightarrow{\mathcal{E}}(t)\right] \psi(\theta, \phi ; t),
$$

where $J$ is the angular momentum operator, $B$ the rotational constant, $\vec{\mu}(\overrightarrow{\mathcal{E}})$ the total dipole moment and $\overrightarrow{\mathcal{E}}$ the electric field. $\theta$ denotes the polar angle between the molecular axis and the polarization direction of the applied field. The motion related to the azimuthal angle $\phi$ can be separated due to cylindrical symmetry. The field-induced dipole is expanded in powers of $\overrightarrow{\mathcal{E}}(t)$,

$$
\vec{\mu}=\vec{\mu}_{0}+\overline{\bar{\alpha}} \cdot \overrightarrow{\mathcal{E}}(t)+\ldots,
$$

where the two first terms (to which our approximation is limited) are respectively the permanent dipole $\vec{\mu}_{0}$ and the polarizability tensor $\overline{\bar{\alpha}}$. We notice that only very intense lasers may require the inclusion in Eq. (12) of higher order terms. The electric field is written as

$$
\overrightarrow{\mathcal{E}}(t)=f(t) \cos (\omega t) \vec{\epsilon}
$$

$\vec{\epsilon}$ being the unit polarization vector, $\omega$ the carrier wave frequency and $f(t)$ the laser pulse envelope. In a highfrequency regime, which is often referred to in models dealing with alignment [2], the permanent dipole interaction, i.e., $\vec{\mu}_{0} \cdot \overrightarrow{\mathcal{E}}(t)$, averages to zero due to the term $\cos (\omega t)$, whereas the polarizability interaction corresponding to $\overline{\bar{\alpha}} \cdot \overrightarrow{\mathcal{E}}^{2}(t)$, involves a $\cos ^{2}(\omega t)$ term which contributes an average $1 / 2$. Finally, in a high-frequency approximation [26, 30], the TDSE [Eq. (11)] is written as

$i \frac{\partial}{\partial t} \psi(\theta, \phi ; t)=\left[B J^{2}-\frac{1}{4} f^{2}(t)\left(\Delta \alpha \cos ^{2} \theta+\alpha_{\perp}\right)\right] \psi(\theta, \phi ; t)$,

where $\Delta \alpha=\alpha_{\|}-\alpha_{\perp}$ is the difference between the parallel $\alpha_{\|}$and perpendicular $\alpha_{\perp}$ components of the polarizability tensor. While excellent alignment can be obtained through adiabatic transport on so-called pendular states 26] resulting from field dressing, only sudden pulses offer the possibility of alignment that persists after the field is over [2]. As opposed to alignment, orientation requires spatial symmetry breaking, and therefore cannot be obtained from a radiative interaction in $\cos ^{2} \theta$ such as the one depicted in Eq. (14) resulting from polarizability. It has recently been shown that very short pulses combining a frequency $\omega$ and its second harmonic $2 \omega$ (in resonance with a vibrational transition), excite, through the combined effect of $\mu_{0}$ and $\alpha$, a mixture of even and odd rotational levels and have the ability to produce post-pulse orientation [31]. But even more efficient orientation is obtained using half-cycle pulses (HCPs), that, through their highly asymmetrical shape, induce a very sudden momentum transfer to the molecule which orients under such a kick after the field is off [28, 32]. It is worth noting that both the $(\omega+2 \omega)$ and the kick mechanisms have 
received a confirmation from optimal control schemes [7]. In a moderate field approximation where the polarizability interaction is neglected as compared to the permanent dipole one, the TDSE which governs the orientation process can be written as

$$
i \frac{\partial}{\partial t} \psi(\theta, \phi ; t)=\left[B J^{2}-f(t) \mu_{0} \cos \theta\right] \psi(\theta, \phi ; t) .
$$

As the two processes (i.e., post-pulse alignment / orientation) require short duration pulses, from now on, we assume a sudden approximation based on the shortness of the pulse duration $\tau$ as compared to the molecular rotational period $T_{\text {rot }}=\pi / B$. For relatively low $j$ (where $j$ labels the eigenstates of $J^{2}$ ), this amounts to the definition of a dimensionless, small perturbative parameter $\varepsilon=\tau B$. This definition, together with a rescaling of time $s=t / \tau$ (such that $s \in[0,1]$ during the pulse) leads to a TDSE that can be treated by time-dependent unitary perturbation theory [33, 34],

$$
i \frac{\partial}{\partial s} \psi(\theta, \phi ; s)=\left[H_{0}-V_{a ; o}(s)\right] \psi(\theta, \phi ; s),
$$

with $H_{0}=\varepsilon J^{2}$ and $V_{a ; o}(s)$ the radiative interaction depending on the process to be described. More precisely, we have for alignment

$$
V_{a}(s)=E_{a}^{2}(s) \cos ^{2} \theta+F_{a}^{2}(s),
$$

and for orientation

$$
V_{o}(s)=E_{o}(s) \cos \theta .
$$

The time-dependent functions are defined as

$$
\begin{aligned}
& E_{a}^{2}(s)=\Delta \alpha \tau f^{2}(\tau s) / 2 \\
& F_{a}^{2}(s)=\alpha_{\perp} \tau f^{2}(\tau s) / 2 \\
& E_{0}(s)=\mu_{0} \tau f(\tau s)
\end{aligned}
$$

Returning now to the successive steps of our approach as detailed in the mathematical setup (Sec. III), we have to define an initial pure quantum state and the observables adequately describing both processes. The initial state of the molecule is taken as the ground state of the rigid rotor,

$$
\left|\psi_{n=0}(t=0)\right\rangle=|j=0, m=0\rangle .
$$

We remark that any other rotational state of the rotor, i.e., $|j \geq m, m\rangle$ could not be taken, by observing that the projection quantum number $m$ is invariant upon the application of linearly polarized pulses. The two observables we consider are $\mathcal{O}_{a}=\cos ^{2} \theta$ for alignment and $\mathcal{O}_{o}=\cos \theta$ for orientation. The goal of the field driven molecular alignment (or orientation) is then to maximize (or to minimize, depending the choice of the orientation) for the largest time duration, the expectation values

$$
\left\langle\mathcal{O}_{a ; o}(s)\right\rangle=\left\langle\psi(\theta, \phi ; s)\left|\mathcal{O}_{a ; o}\right| \psi(\theta, \phi ; s)\right\rangle
$$

after the pulse is over.

The second step consists in the reduction of the dimensionality of the infinite physical Hilbert space by considering a finite subspace $\mathcal{H}_{m=0}^{(N)}$ generated by the first $N$ eigenvectors of $J^{2}$, i.e., $|j, m=0\rangle(j=0,1, \ldots, N-1)$. The observables are projected in this subspace according to

$$
\mathcal{O}_{a ; o}^{(N)}=P_{m=0}^{(N)} \mathcal{O}_{a ; o} P_{m=0}^{(N)}
$$

with the projectors explicitly given by

$$
P_{m=0}^{(N)}=\sum_{j=0}^{N-1}|j, m=0\rangle\langle j, m=0| .
$$

It is clear from Eq. (24) that $\mathcal{O}_{a ; o}^{(N)}$ has, as opposed to $\cos ^{2} \theta$ or $\cos \theta$, a purely discrete spectrum. The target states $\left|\chi_{a ; o}^{(N)}\right\rangle$ which maximize the alignment (or the orientation) in the subspace $\mathcal{H}_{m=0}^{(N)}$ are simply the eigenstates of $\mathcal{O}_{a ; o}^{(N)}$ with the highest eigenvalue. Their calculation in terms of the appropriate expansion coefficients $c_{j}^{a ; o}$ in the $|j, m=0\rangle$ basis,

$$
\left|\chi_{a ; o}^{(N)}\right\rangle=\sum_{j=0}^{N-1} c_{j}^{a ; o}|j, m=0\rangle,
$$

involves the diagonalization of the $(N \times N)$ matrices of $\mathcal{O}_{a ; o}^{(N)}[\mathrm{Eq}$. (24)] written in the same basis. For instance, in the simplest case of orientation, a tridiagonal matrix of the operator $\mathcal{O}_{o}^{(N)}$ results (when using the approximation $\langle j, m=0|\cos \theta| j \pm 1, m=0\rangle=1 / 2$, valid for $j \gg m$ ) in the analytical expression

$$
\left|\chi_{o}^{(N)}\right\rangle \simeq\left(\frac{2}{N+1}\right)^{1 / 2} \sum_{j=0}^{N-1} \sin \left(\pi \frac{j+1}{N+1}\right)|j, m=0\rangle
$$

with the corresponding maximal orientation found in this subspace

$$
\left\langle\chi_{o}^{(N)}|\cos \theta| \chi_{o}^{(N)}\right\rangle \simeq \cos \left(\frac{\pi}{N+1}\right)
$$

Having determined the kinematical constraints, we can now analyze the dynamical realizability of these target states. This kind of Hamiltonian (involving a dipole interaction with the control field) has already been investigated by S. G. Schirmer and co-workers [12] and it was shown that the Lie algebra generated by $i H_{0}^{(N)}$ and $i \mathcal{O}_{a ; o}^{(N)}$ is $u(N)$ (Theorem 1 of Ref. [12]). The system is therefore completely controllable.

The next step consists in applying our control strategy. For doing so, we determine the evolution operator for individual pulses. This is done in the impulsive sudden limit [28, 29]. The interaction term of the alignment process $V_{a}(s)$ [Eq. (17)] involves the square of the electric 
field amplitude. The time evolution can be expressed in terms of a free evolution combined with

$$
U_{a}=\exp \left[i A_{a} \cos ^{2} \theta\right],
$$

where

$$
A_{a}=\int_{0}^{1} E_{a}^{2}(s) d s
$$

is the total pulse-square area. We note that we have not taken into account the $\theta$-independent contribution $F_{a}^{2}(s)$ to $V_{a}(s)$ as it would only contribute a pure phase factor. For the case of orientation, we get as the instantaneous evolution operator describing the kick

$$
U_{o}=\exp \left[i A_{0} \cos \theta\right],
$$

with

$$
A_{o}=\int_{0}^{1} E_{o}(s) d s
$$

the total pulse area. Between pulses, the molecule evolves under the effect of its field-free rotation, described by the evolution operator

$$
U_{H_{0}}=\exp \left[-i \varepsilon J^{2} s\right] .
$$

One can easily check the necessary commutation relations

$$
\left[\mathcal{O}_{a ; o}^{(N)}, U_{a ; o}\right]=0
$$

implying that the target states $\left|\chi_{a ; o}^{(N)}\right\rangle$ are eigenfunctions of both $\mathcal{O}_{a ; o}^{(N)}$ and $U_{a ; o}$. Note that all quantities $(s, \varepsilon$, $\left.E_{a}^{2}, E_{o}\right)$ are dimensionless, and hence do not depend on a specific molecular system. Times can be expressed in terms of fractions of the molecular rotational period $T_{\text {rot }}$. Moreover, the molecule-laser interaction characteristics $\left(E_{a}^{2}\right.$ or $\left.E_{o}\right)$ combine the field strength $\mathcal{E}$ with molecular constants $\alpha$ or $\mu_{0}$, in such a way that the same values may be considered for different molecules at other, adequately chosen laser intensities. The final step for the control scheme consists in applying the instantaneous perturbations at specific times. More precisely, as has been discussed in the mathematical setup (Sec. III), two distinct strategies are adopted for the determination of these times. The first one $(S 1)$ consists in taking the series of $s_{i}$ when $\left\langle\mathcal{O}_{a ; o}^{(N)}\right\rangle(s)$ reaches its successive maxima (global or local) during the free evolution following a kick. The second one $(S 2)$ consists in taking the $s_{i}$ 's as times when the modulus of the projection of the wave function on the target states $\left|\left\langle\psi_{\lambda}(s) \mid \chi_{a ; o}^{(N)}\right\rangle\right|$ reaches a maximum during the free evolution. The numerical results obtained by both strategies are presented in Sec. IV

We now discuss the relative merits of the two schemes from a theoretical point of view. For the strategy $S 1$, the slope after the pulse can be explicitly determined in the infinite-dimensional case, as shown in Appendix [C]

$$
\left.\frac{d}{d s}\langle\cos \theta\rangle\right|_{s_{i}+0}=2 \varepsilon A\left(\mathbf{1}-\left\langle\cos ^{2} \theta\right\rangle\right),
$$

for orientation, and

$$
\left.\frac{d}{d s}\left\langle\cos ^{2} \theta\right\rangle\right|_{s_{i}+0}=2 A \varepsilon\left\langle\sin ^{2} 2 \theta\right\rangle,
$$

for alignment. As $\left\langle\cos ^{2} \theta\right\rangle<1$ and $\left\langle\sin ^{2} 2 \theta\right\rangle>0$, one deduces that, in both cases, the slope takes a non-zero value after the application of the kick, whatever the pulse area $A$ of the field. This point proves that the control scheme can be iterated for the alignment / orientation processes. In the finite dimensional case, the corresponding expression for orientation derived in Appendix $[$ is slightly different. We obtain for $\cos ^{(N)} \theta$

$$
\left.\frac{d}{d s}\left\langle\cos ^{(N)} \theta\right\rangle\right|_{s_{i}+0}=2 \varepsilon A\left(\mathbf{1}-\left\langle\left(\cos ^{(N)} \theta\right)^{2}\right\rangle+B\right)+O\left(A^{2}\right),
$$

$B$ being a boundary term which is of the form

$$
B=-\frac{N^{2}+2 N+1}{2 N+1}\left|a_{N}\right|^{2},
$$

where the $a_{n}$ 's are the coefficients of the wave function in the basis $|0 \leq j \leq N-1, m=0\rangle$. Here, we notice that we cannot conclude as above and arguments of dimensionality have to be used. In this way, Appendices $\mathrm{A}$ and $\mathrm{B}$ show that the unique fixed point of strategy $S 2$ is the target state $\left|\chi^{(N)}\right\rangle$ whereas scheme $S 1$ can possess several fixed points as the eigenfunctions of the operator $\mathcal{O}^{(N)}$. However, the strategy $S 1$ has the advantage that the target state can be selected during the dynamical process by appropriately choosing the intensities and the number of laser pulses. This is not the case for the scheme $S 2$ because the target state has to be selected a priori.

\section{RESULTS}

The results are presented in a general way, transferable to any particular molecule (characterized by its rotational period, its permanent dipole and its polarizability) interacting with any pulsed field (characterized by its moderate amplitude and its short duration as compared to the molecular rotational period). The relevant parameters which gather both molecule and field constants are taken to be $A_{a}=1.5, A_{o}=1$, and $\varepsilon=0.03$. They are compatible with any molecule, provided that the field amplitude is adequately chosen. For $\mathrm{LiCl}$, for instance, this amounts to a pulse duration of about 0.3 ps and a field amplitude of $1.5 \times 10^{5} \mathrm{~V} \mathrm{~cm}^{-1}[28]$ in the case of the orientation. Time is indicated in fractions of the molecular rotational period. The presentation of the results follows the different steps of the control strategy. Analytical estimations of the dynamical parameters are reported in Appendix D. 


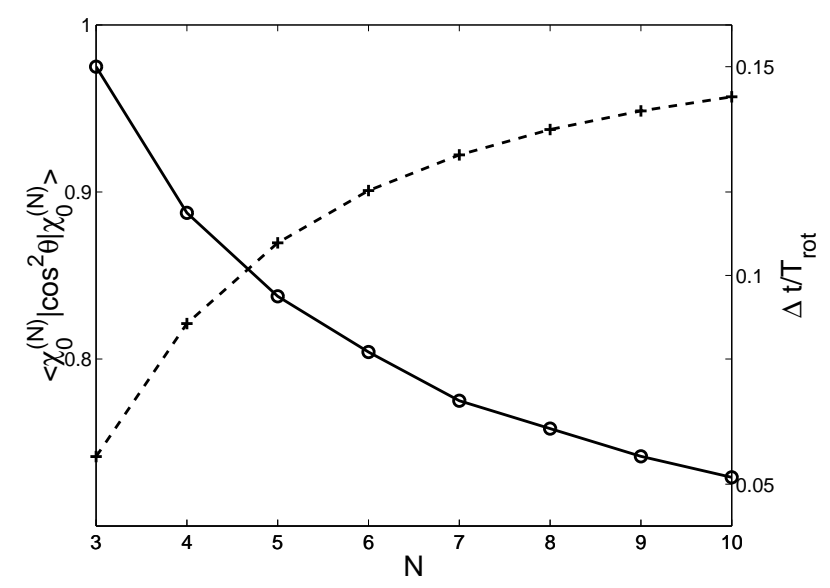

FIG. 1: Maximum alignment efficiency (crosses) and associated duration (open circles) as a function of $N$, the dimension of the rotationally excited subspace $\mathcal{H}_{m=0}^{(N)}$ (see text). The solid and dashed lines are just to guide the eye.

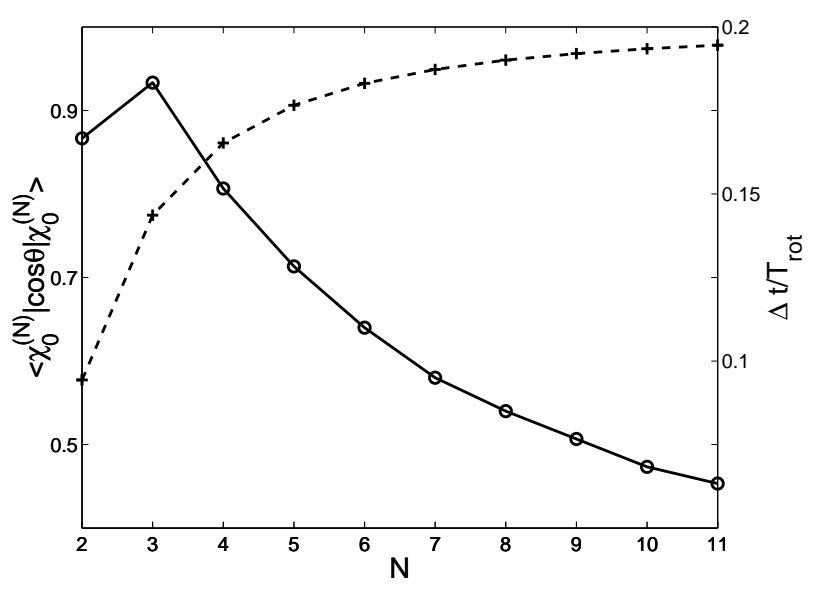

FIG. 2: Same as Fig 1 but for orientation.

\section{A. The finite dimensional subspace}

Relevant information for the characterization of alignment and orientation in the finite subspace $\mathcal{H}_{m=0}^{(N)}$ as a function of its dimensionality $N$ are displayed in Figs. 1 and 2] The maximum efficiency (approximately given by Eq. (28) for the case of orientation) that can ideally be expected for a process that stays confined within $\mathcal{H}_{m=0}^{(N)}$ is given as $\left\langle\chi_{0}^{(N)}\left|\mathcal{O}_{a ; o}^{(N)}\right| \chi_{0}^{(N)}\right\rangle$. The maximum duration, measuring the relative duration of the alignment / orientation processes over which $\left\langle\mathcal{O}_{a ; o}^{(N)}(s)\right\rangle$ remains larger than 0.5 during the field-free evolution, is indicated in terms of a fraction of the rotational period as $\Delta t / T_{\text {rot }}$. The information displayed is crucial for the choice of the dimensionality $N$ of the reduced subspace: a larger $N$ is more suitable for a better efficiency of alignment / orientation, but leads to a shorter duration. The compromise between maximum efficiency and duration is to be done at that step. In order to keep an alignment / orientation duration of the order of one tenth of the rotational period (amounting to durations exceeding $10 \mathrm{ps}$ for heavy diatomic molecules such as NaI), $N$ has to be limited to 5, as can be observed from Figs. 1]and 2] This may seem rather limiting in view of the moderate molecular rotational excitation, but even such a low dimension $(N=5)$ turns out to be sufficient for very efficient alignment (about 0.85 ) or orientation (about 0.9). This fixes completely the target states (approximately given by Eq. (27) in the case of orientation) for both processes.

\section{B. Dynamics controlled by the two strategies}

The two strategies, noted $S 1$ and $S 2$, can be exploited in the same way, the time delays between the successive pulses being defined following one of the two processes (maxima of $\left\langle\mathcal{O}_{a ; o}^{(5)}\right\rangle$ for $S 1$, or maxima of $\left|\left\langle\chi_{a ; o}^{(5)} \mid \psi_{\lambda}(t)\right\rangle\right|^{2}$ for $S 2)$. We look for the dynamical behavior of the two quantities, namely the expectation value of the observables $\left\langle\mathcal{O}_{a ; o}^{(5)}\right\rangle$ and the projections on the target states $\left|\left\langle\chi_{a ; o}^{(5)} \mid \psi_{\lambda}(t)\right\rangle\right|^{2}$. All results are displayed with the first pulse taken as the origin of time and extend at least one rotational period $T_{\text {rot }}$ after the last pulse. This helps to show the complete field-free behavior of the dynamics, which repeats periodically leading to revival structures. Figures 3] and 4 illustrate the results of applying strategy $S 1$ to the alignment and orientation processes, respectively. Noticeably different behavior is obtained for the two processes: clearly, a smaller number of pulses is necessary to control alignment (i.e., 6 pulses for alignment instead of 15 for orientation). The first pulse already provides an alignment efficiency of 0.75 , whereas it only yields 0.5 orientation efficiency. Conversely, the overall dynamics (conditioning the delays between successive pulses) is much more oscillatory with complex structures in the case of alignment as compared to orientation where it progressively increases up to 0.89 , which is almost the optimal limit as found from Fig. 2. In all cases, a comparison of the observables and the projections calculated using the exact wave function $\psi_{\lambda}(s)$ with the one propagated in the subspace $\mathcal{H}_{m=0}^{(5)}$ supports the claim that the rotational dynamics actually resides within $\mathcal{H}_{0}^{(5)}$. In both cases, panels (b) shows the way the wave function $\psi_{\lambda}(s)$ gets close to the target state $\chi_{a ; o}^{(5)}$, illustrating the successful outcome of the control strategy with a coherent choice of parameters $A, N$, and the number of pulses for appropriately describing the dynamics. Finally, the post-pulse dynamics leads to results particularly remarkable with respect to previous proposals. An efficiency of about 0.85 with a duration of $(1 / 10)$ th of the rotational period is achieved for alignment, with even better results for orientation, i.e., 0.89 efficiency and (2/10)th of the rotational period duration (which corresponds to about $2 \mathrm{ps}$ for a light molecule like $\mathrm{LiCl}$ and $20 \mathrm{ps}$ for a heavy one like $\mathrm{NaI}$ ). 

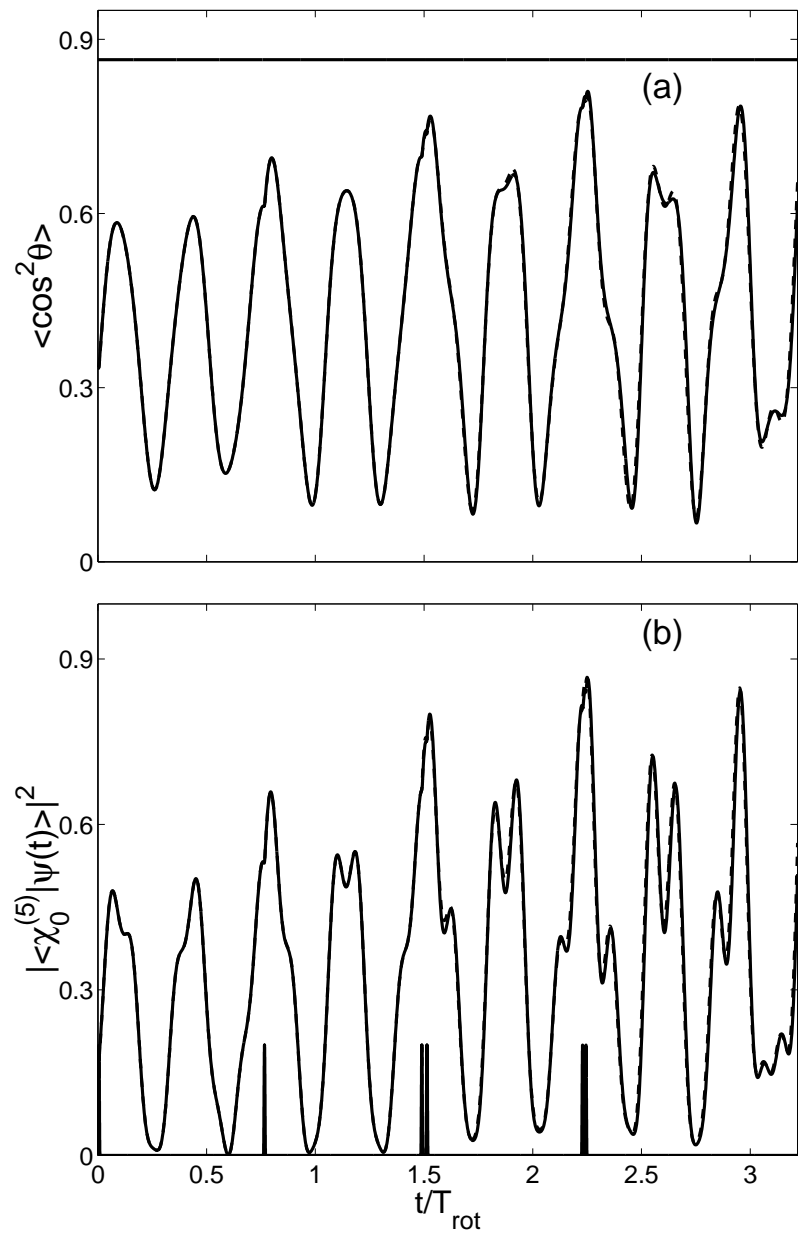

FIG. 3: Alignment dynamics during and after the train of pulses determined by strategy $S 1$ of perturbing at the global maxima of $\left\langle\cos ^{2} \theta\right\rangle$ : panel (a) for $\left\langle\psi_{\lambda}(s)\left|\cos ^{2} \theta\right| \psi_{\lambda}(s)\right\rangle$ and panel (b) for $\left|\left\langle\chi_{a}^{(5)} \mid \psi_{\lambda}(s)\right\rangle\right|^{2}$. The solid line corresponds to the exactly propagated wave function $\psi_{\lambda}(s)$ and the dashed line to the propagation of $\psi_{\lambda}(s)$ in the subspace $\mathcal{H}_{m=0}^{(5)}$. The train of pulses is displayed on panel (b) and the optimal alignment in $\mathcal{H}_{m=0}^{(5)}$ is indicated by the horizontal line on panel $(a)$.

We next notice a series of local maxima in Fig. 3 (for instance, just after the first kick), which could be used for the control strategy. This point is illustrated in Fig. 5 for strategy $S 1$. It turns out that excellent results are obtained for the alignment process using in the control scheme the local maxima (the next one after the application of the pulse). More unexpectedly, this leads to a better result than the use of the global maxima, as optimal alignment is almost achieved after only 4 kicks (while 6 are used in Fig. (3). In this example, it is clear that the best choice for the convergence of the process is to kick at a local maximum. However, we did not manage to establish general rules for this degree of freedom and it thus seems that numerical tests have to be undertaken for each practical case.

The outcome of the calculation when applying strategy
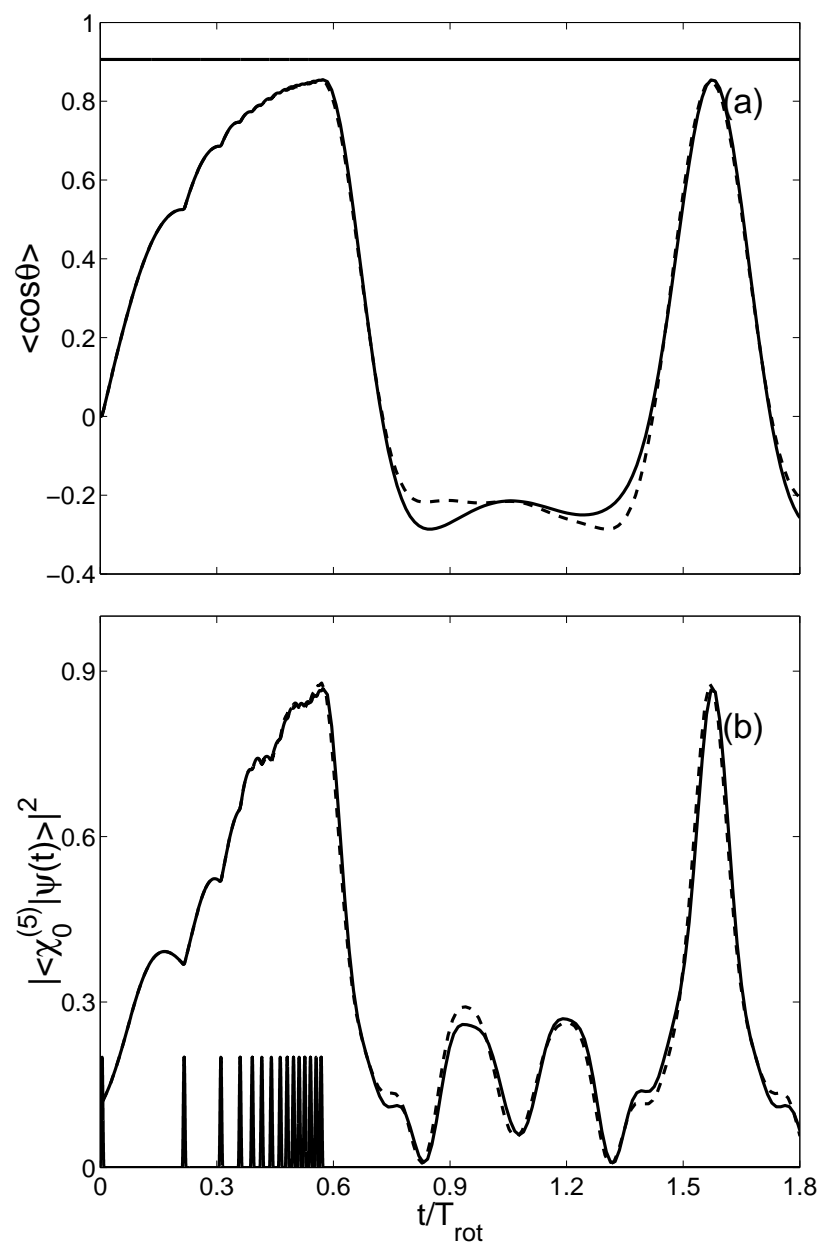

FIG. 4: Same as Fig. 3 but for the orientation dynamics.

$S 2$ to the orientation, taken as an example, is displayed in Fig. 6. The results obtained are very similar to those using strategy $S 1$, with a smaller number of pulses (viz., 9) but leading to slightly less efficient orientation. This suggests a great similarity between the two strategies from a numerical point of view, as can be seen when comparing Figs. 4 and 6

\section{Robustness}

The robustness of the overall strategy has in principle to be checked against two variables: the time delays between successive pulses and the total energy delivered by individual pulses. Figure 7 illustrates the robustness against the most sensitive parameter, i.e., the time delays $s_{i}$, where $\left\langle\mathcal{O}_{o}^{(N)}\left(s_{i}\right)\right\rangle$ is maximum. This is given for the orientation process only, by considering again the two strategies $S 1$ and $S 2$. Strategy $S 2$ seems here more robust, but this is only due to the smaller number of pulses which are necessary for reaching the target state (9 for $S 2$, instead of 15 for $S 1$ ).

As for experimental feasibility, the accuracy of the light 

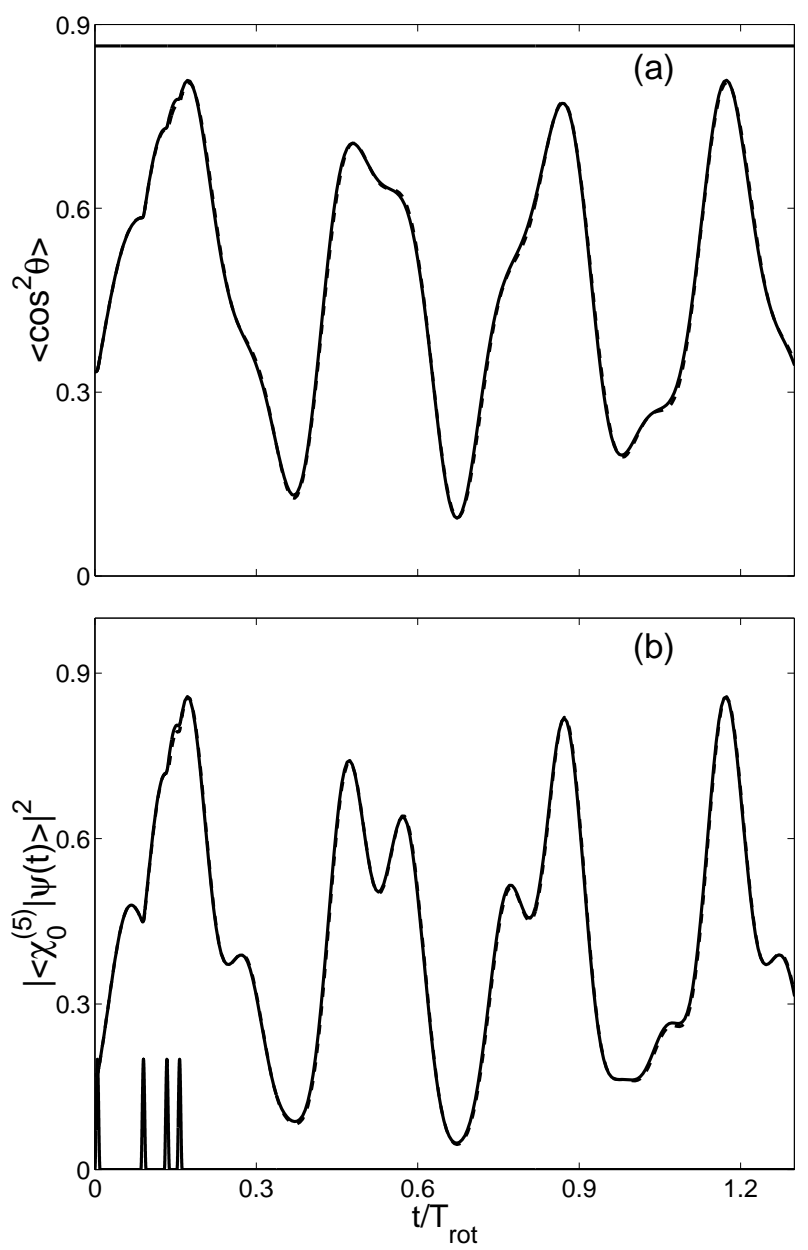

FIG. 5: Same as Fig. 3 but local maxima (the first maximum after the pulse) have been used.

paths followed by successive pulses is of the order of $0.2 \mu \mathrm{m}$, corresponding to $0.6 \mathrm{fs}$. This is, for instance for a light diatomic molecule like $\mathrm{LiCl}$, less than 1/10th of the inaccuracies considered when varying the time delays, in Fig. 7 In other words, both strategies are very robust with respect to such inaccuracies affecting the moleculepulse interaction terms. On the contrary, the measure of the total energy per pulse is affected by a more severe inaccuracy. This is checked in Fig. 8 by varying $A$ by $10 \%$, for both strategies $S 1$ and $S 2$. Remarkable robustness is achieved, advocating for an experimental feasibility of the control scheme: a few short pulses (say 5 to 6 ), with a moderate total energy determined within $10 \%$ of accuracy, applied with time delays of the order of 1 ps with an accuracy of $1 \mathrm{fs}$, leads to an excellent control of the orientation for any molecular system.

\section{CONCLUSIONS}

In this work, we have developed a laser control strategy for a molecular system initially in a pure quantum
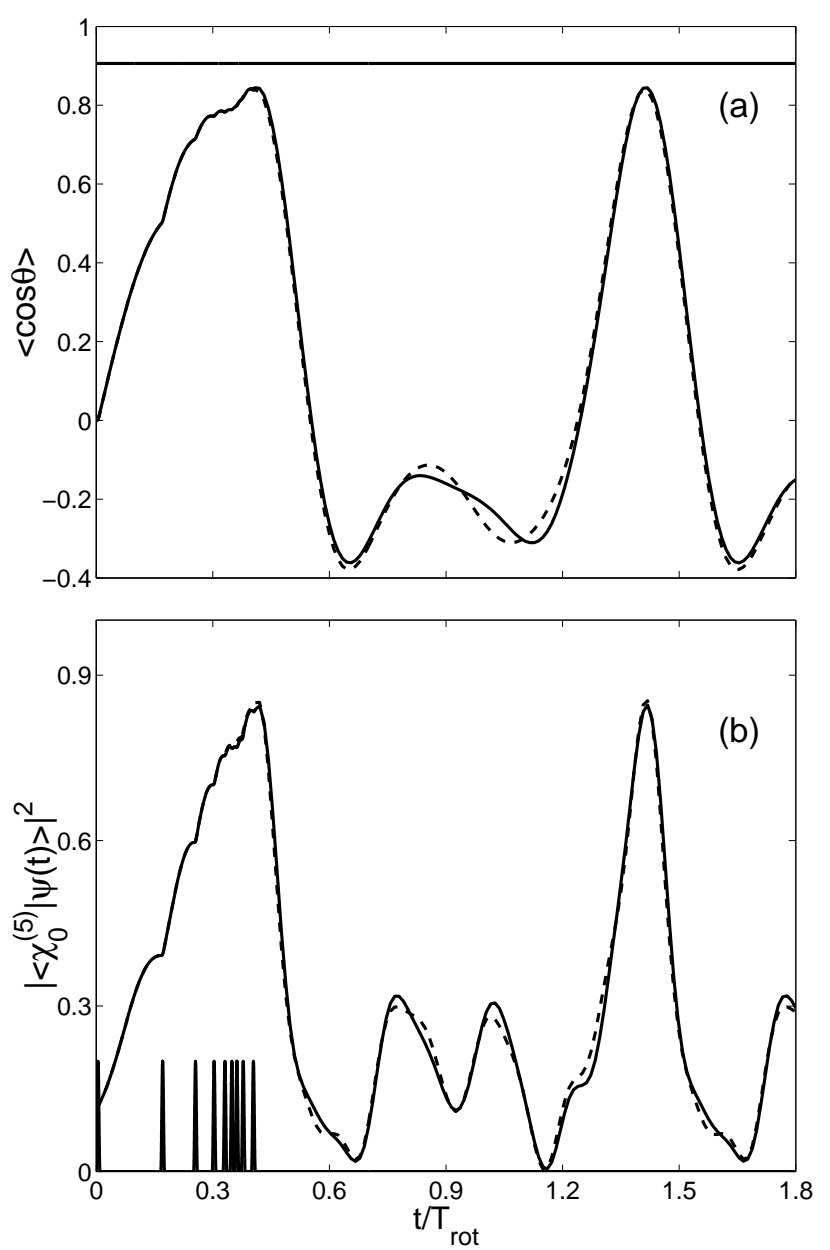

FIG. 6: Same as Fig. 4 but referring to strategy $S 2$, perturbing the system at the maxima of $\left|\left\langle\chi_{o}^{(5)} \mid \psi_{\lambda}(s)\right\rangle\right|^{2}$.

state, aiming at the maximalization (or minimalization) of an observable $\mathcal{O}$ given by an operator, not commuting with the molecular field-free Hamiltonian. The strategy consists of four steps: reduction of the dimensionality of the original physical Hilbert space; definition of a target state as an eigenvector of the reduced observable in the finite subspace; analysis of the controllability of the system; application of a train of sudden perturbations $U$, commuting with $\mathcal{O}$, at times where the expectation value of $\mathcal{O}$ reaches a maximum during its field-free evolution. A process similar in its spirit consists in applying the pulses at times when the projection of the time-evolved wave function on the target states reaches maximum values. This latter strategy yields comparable numerical results even if, from a theoretical point of view, its set of fixed points (which corresponds only to the target state) can be smaller than (or equal to) the one of the first process. Moreover, it is worth noting that such general strategies are not only independent of the particular molecule, but are also transferable to different control issues and different observables. Finally, we have checked the robustness with respect to inaccuracies in the time delays between 

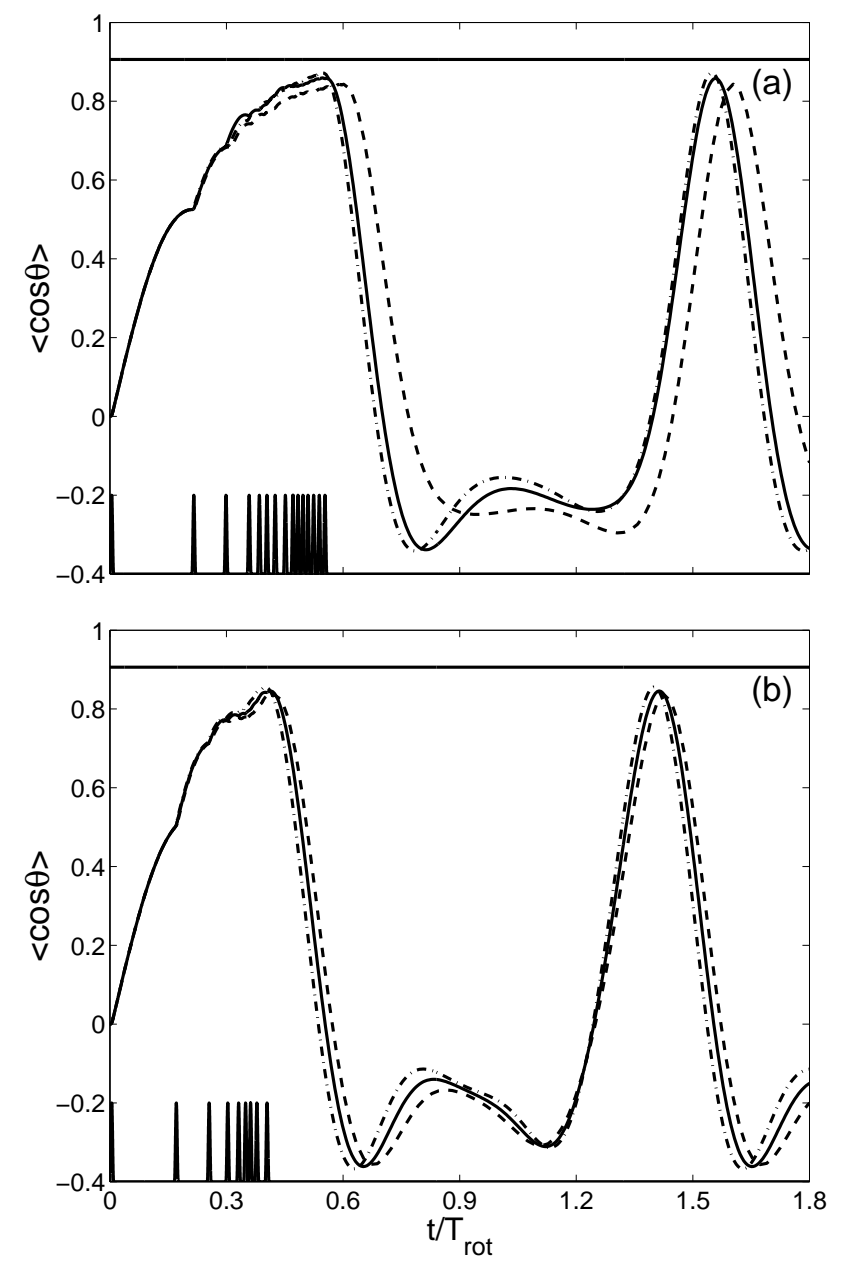

FIG. 7: Robustness of the orientation dynamics when shifting the time delays between pulses systematically by $\pm 0.1 \%$ of the rotational period (the dashed line corresponds to overestimates by $+0.1 \%$ whereas the dotted-dashed one to underestimates by $-0.1 \%$, the solid line corresponding to the calculation of Fig. (4). The strategy $S 1$ is displayed on panel (a) and $S 2$ on panel (b).

pulses or the total pulse energies, and found it to be remarkably good, as long as moderate intensity and short radiative interactions are considered.

Control strategies based on pure states are however subject to important modifications when dealing with temperature effects. Orientation, for instance, drastically decreases for increasing temperatures [32, 35, 36, 37]. This is basically due to the fact that, at non-zero temperature, the initial state is in general not a pure state, but a mixed one, i.e., a superposition of a statistical ensemble of rotational states with $m \neq 0$, which tend to misalign the molecule. The dynamics of such mixed states is described by a density operator $\rho(t)$ (instead of a wave function) evolving according to the von Neuman equation (instead of the Schrödinger equation). Following the dimensionality reduction of the Hilbert space, the control strategy looks now for the optimal density operator $\rho^{(N)}$
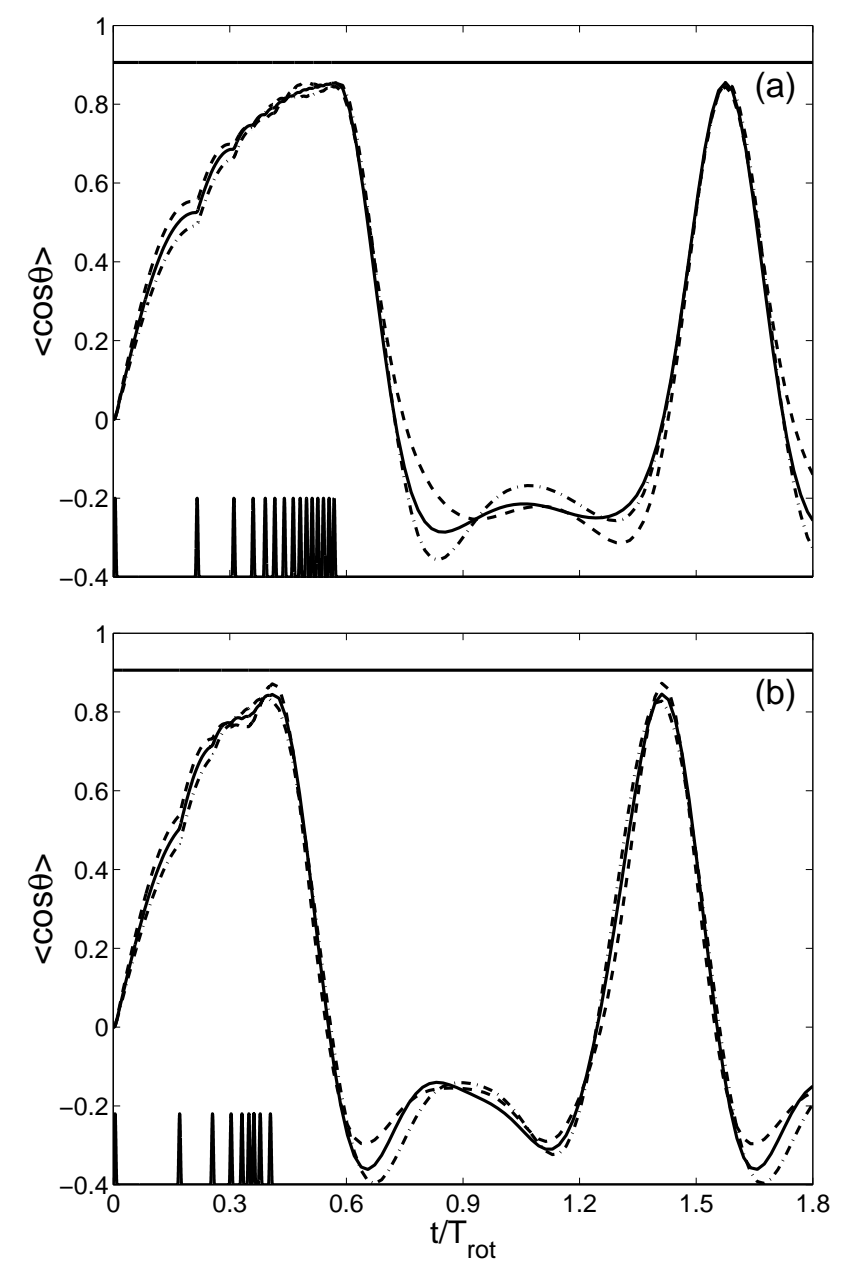

FIG. 8: Robustness of the orientation dynamics varying the pulse energy by $10 \%$. The full line corresponds to $A=1$, i.e., the calculation of Fig. 4 the dotted and dotted-dashed lines are for $A=1.1$ and $A=0.9$, respectively. The strategy $S 1$ is displayed on panel (a) and $S 2$ on panel (b).

which maximizes the observable $\mathcal{O}^{(N)}$ in the same subspace. This amounts to maximizing $\operatorname{Tr}\left[\rho^{(N)} \mathcal{O}^{(N)}\right]$, with the commutation relation $\left[\rho^{(N)}, \mathcal{O}^{(N)}\right]=0$. It has been shown 9,10$]$ that such a goal can be achieved by an unitary operator $U$ that appropriately orders the common eigenvectors of $\rho^{(N)}$ and $\mathcal{O}^{(N)}$, such that the one corresponding to the highest eigenvalue of $\rho^{(N)}$ be associated with the one corresponding to the highest eigenvalue of $\mathcal{O}^{(N)}$, and so forth. An even more sophisticated generalization of the control strategy is to be looked for when the particular system under consideration evolves while interacting with a physical environment describing collisional processes in a gas, or friction in a liquid [38, 39]. The strategies that have to be worked out would involve non-unitary perturbations $U$ and a special attention has to be paid to decoherence [40, 41]. Work in these directions is in progress. 


\section{Acknowledgments}

Support from the Conseil Régional de Bourgogne is gratefully acknowledged.

\section{APPENDIX A: ANALYSIS OF THE SET OF FIXED POINTS: THE GENERAL CASE}

The goal of this appendix is to determine the set of fixed points of the control strategy $S 1$. Indeed, as mentioned in Sec. III a crucial question for the convergence of the control scheme is the set $\mathcal{F}$ of its fixed points. In general, we are not able to exactly determine $\mathcal{F}$ but only a larger set $\mathcal{S}$. The latter contains the wave functions $\left|\psi_{s}\right\rangle$ which fulfill the requirements

$$
\left\langle\psi_{s}\left|\left[H_{0}, \mathcal{O}^{(N)}\right]\right| \psi_{s}\right\rangle=0
$$

and

$$
\left\langle\psi_{s}\left|U_{\tilde{A}}^{-1}\left[H_{0}, \mathcal{O}^{(N)}\right] U_{\tilde{A}}\right| \psi_{s}\right\rangle=0,
$$

for all values of the real parameter $\tilde{A}$. As mentioned above, we can show that $\mathcal{F}$ is a subset of $\mathcal{S}$. For the purpose of proving this result, we observe that just before the pulse at $t_{i}-0$ one has

$$
\left.\frac{d}{d t}\left\langle\mathcal{O}^{(N)}\right\rangle\right|_{t_{i}-0}=\left.i\left\langle\left[H_{0}, \mathcal{O}^{(N)}\right]\right\rangle\right|_{t_{i}-0}=0
$$

whereas just after the pulse at $t_{i}+0$

$$
\begin{aligned}
\left.\frac{d}{d t}\left\langle\mathcal{O}^{(N)}\right\rangle\right|_{t_{i}+0} & =\left.i\left\langle U_{\tilde{A}}^{-1}\left[H_{0}, \mathcal{O}^{(N)}\right] U_{\tilde{A}}\right\rangle\right|_{t_{i}+0} \\
& =\left.i\left\langle\left[U_{\tilde{A}}^{-1} H_{0} U_{\tilde{A}}, \mathcal{O}^{(N)}\right]\right\rangle\right|_{t_{i}+0}
\end{aligned}
$$

We can conclude by arguing that if there exists a value of the parameter $\tilde{A}$ such that the slope undergoes a change from zero [Eq. (A3)] to a non-zero finite value [Eq. (A4)], then $\left\langle\mathcal{O}^{(N)}(t)\right\rangle$ will reach within the period $T$ a maximum strictly larger than the one obtained prior the application of the pulse.

We are now in a position to state the following theorem: $\mathcal{S}=\left\{\left|\chi^{(n)}\right\rangle\right\}$ (the $\left|\chi^{(n)}\right\rangle$ 's being the eigenstates of $\left.\mathcal{O}^{(N)}\right)$ if and only if the dimension of the vector space $\mathcal{V}$ generated by $\left[H_{0}, \mathcal{O}^{(N)}\right]$ and the elements of the form $U_{\tilde{A}}^{-1}\left[H_{0}, \mathcal{O}^{(N)}\right] U_{\tilde{A}}$, where $\tilde{A} \in \mathbb{R}$, is equal to $N(N-1)$. The proof goes as follows.

We first recall that the complete controllability of the system is assumed. This condition is equivalent to requiring that the Lie algebra $\mathcal{L}$ generated by $i H_{0}$ and $i H_{I}$ is $u(N)$ or that the dimension of this vector space is $N^{2}$. Moreover, $U_{\tilde{A}}^{-1}\left[H_{0}, \mathcal{O}^{(N)}\right] U_{\tilde{A}}$ being a skew-hermitian operator, it can be shown that $\mathcal{V}$ is a subspace of $\mathcal{L}$. The starting point for the following computations will be the standard basis of $u(N)$ [12],

$$
\left\{\begin{array}{l}
x_{m n}=e_{m n}-e_{n m}, \\
y_{m n}=i\left(e_{m n}+e_{n m}\right) \\
d_{n n}=i e_{n n}
\end{array}\right.
$$

where $e_{m n}=\left|\chi^{(m)}\right\rangle\left\langle\chi^{(n)}\right|, 1 \leq n \leq N-1$ and $n<$ $m \leq N$. We denote $D$ the dimension of $\mathcal{V}$. This vector space is generated by the set $v_{k}(k=1, \ldots, D)$, which can be written as a linear combination of elements of the basis given by Eq. A6 . Expanding $\left[H_{0}, \mathcal{O}^{(N)}\right]$ and $U_{\tilde{A}}^{-1}\left[H_{0}, \mathcal{O}^{(N)}\right] U_{\tilde{A}}$ with respect to the previous set $v_{k}$ and taking the mean value of these operators over a wave function $\left|\psi_{s}\right\rangle \in \mathcal{S}$, we obtain a system of linear equations. Inverting the system, one gets $D$ equations of the form

$$
\left\langle\psi_{s}\left|v_{k}\right| \psi_{s}\right\rangle=0
$$

As $\left|\chi^{(n)}\right\rangle \in \mathcal{S}$ and $\left\langle\chi^{(n)}\left|d_{n n}\right| \chi^{(n)}\right\rangle=1$ for any $n=$ $1, \ldots, N$, it is clear that $\mathcal{V}$ does not contain any linear combination of the $d_{n n}$ 's, which shows that the dimension of $\mathcal{V}$ is at most $N(N-1)$. Hence, if we assume that, for instance, $x_{m n} \in \mathcal{V}$ and $y_{m n} \in \mathcal{V}$, we obtain by introducing the wave function $|\psi\rangle=\sum_{n=1}^{N} c_{n}\left|\chi^{(n)}\right\rangle$ into Eq. A7

$$
\left\{\begin{array}{l}
c_{m}^{*} c_{n}-c_{n}^{*} c_{m}=0, \\
c_{m}^{*} c_{n}+c_{n}^{*} c_{m}=0 .
\end{array}\right.
$$

From Eqs. (A8), one deduces that $c_{m}=0$ or $c_{n}=0$. By repeating this procedure for each couple $(m, n)$, it can be shown that the states $\left|\chi^{(n)}\right\rangle$, for which for some $n_{0}$

$$
\left\{\begin{array}{l}
c_{n_{0}}=1, \\
c_{m ; m \neq n_{0}}=0
\end{array}\right.
$$

are the unique elements of $\mathcal{S}$ if and only if the dimension of $\mathcal{V}$ is $N(N-1)$. Numerical calculations of this dimension and complementary results are presented in Appendix B] Finally, a conclusion regarding this theorem is that if the dimension of $\mathcal{V}$ is $N(N-1)$, then the set $\mathcal{F}$ of fixed points of the control strategy is a subset of $\left\{\left|\chi^{(n)}\right\rangle\right\}$, the set of eigenvectors of the impulsive propagator $U_{\tilde{A}}$, which are also the ones of $\mathcal{O}^{(N)}$ as the two operators commute.

\section{APPENDIX B: ANALYSIS OF THE SET OF FIXED POINTS IN THE SUDDEN APPROXIMATION}

This appendix particularizes the general arguments of the Appendix A to the sudden approximation. We first consider the strategy $S 1$. In this case, we have to determine the dimension of the vector space $\mathcal{V}$ defined in Appendix $\mathrm{A}$ In the sudden approximation, we recall that the evolution operator $U_{\tilde{A}}$ can be written as [Eq. (10)]

$$
U_{\tilde{A}}=e^{i \tilde{A} H_{I}},
$$


and $\mathcal{V}$ is then generated by the elements of the form $e^{-i \tilde{A} H_{I}}\left[H_{0}, \mathcal{O}^{(N)}\right] e^{i \tilde{A} H_{I}}$, where $\tilde{A} \in \mathbb{R}$. For the sake of clarity, we now consider that $H_{I}=\mathcal{O}^{(N)}$ (this assumption is fulfilled for the alignment / orientation processes). Using the Campbell-Hausdorf formula [42, simple algebra shows that $\mathcal{V}$ is also spanned by the operators $a d^{n}\left(H_{0}, \mathcal{O}^{(N)}\right)(n \geq 1)$. The notation $a d^{n}(B, C)$ for the operators $B$ and $C$ is defined by the recurrence formula

$$
a d^{n}(B, C)=\left\{\begin{array}{ll}
B & n=0 \\
{\left[a d^{n-1}(B, C), C\right]} & n \geq 1
\end{array} .\right.
$$

From a practical point of view, owing to the complexity of analytical calculations, a numerical algorithm, which closely follows the one for complete controllability [43], can be used to determine the dimension of $\mathcal{V}$. The first step consists in rewriting the matrix of each operator $a d^{n}\left(H_{0}, \mathcal{O}^{(N)}\right)(n \geq 1)$ as an $N^{2}$ column vector obtained by concatenating its columns. We next construct a new matrix $R$ with these vectors, each vector corresponding to a column of $R$. Finally, the dimension of $\mathcal{V}$ is given by the rank of $R$.

We apply this method to the orientation process in a finite dimensional space. The results of these computations are presented in Tab. I where the notation $\operatorname{dim}(\mathcal{V})$ corresponds to the dimension of the vector space $\mathcal{V}$. For

TABLE I: Dimensions of the vector spaces $\mathcal{L}$ and $\mathcal{V}$ as a function of $N$ for the orientation dynamics. The third column indicates the maximum of the dimension of $\mathcal{V}$.

\begin{tabular}{c|c|c|c}
\hline $\mathrm{N}$ & $\operatorname{dim}(\mathcal{L})$ & Max. of $\operatorname{dim}(\mathcal{V})$ & $\operatorname{dim}(\mathcal{V})$ \\
\hline \hline 3 & 9 & 6 & 4 \\
4 & 16 & 12 & 8 \\
5 & 25 & 20 & 12 \\
\hline
\end{tabular}

the orientation, as $\operatorname{dim}(\mathcal{V})<N(N-1)$, one deduces that $\mathcal{S} \neq\left\{\left|\chi^{(n)}\right\rangle\right\}$. This point can also be understood through the following calculation.

We introduce the operator $C=\left[H_{0}, \mathcal{O}^{(N)}\right]$ and we note $C_{m n}$ its matrix elements in the basis $\left\{\left|\chi^{(n)}\right\rangle\right\}$,

$$
C_{n m}=\left\langle\chi^{(n)}|C| \chi^{(m)}\right\rangle .
$$

For operators $H_{0}=J^{2}$ and $\mathcal{O}^{(N)}=\cos ^{(N)} \theta$, it can furthermore be checked that $C_{m n}=0$ if $m=n$ and $C_{m n} \neq 0$ otherwise. Assuming that the slope is zero before and after the pulse, we can easily show that

$$
\left\{\begin{array}{l}
\sum_{m, n=1}^{N} c_{m}^{*} c_{n} C_{m n}=0, \\
\sum_{m, n=1}^{N} e^{i \widetilde{A}\left(\chi_{m}-\chi_{n}\right)} c_{m}^{*} c_{n} C_{m n}=0,
\end{array}\right.
$$

where the $c_{n}$ 's are the weighting coefficients of the wave function just before the pulse in the basis of the eigenstates of $\mathcal{O}^{(N)}$, and the $\chi_{n}$ 's the eigenvalues of $\mathcal{O}^{(N)}$. From Eqs. (B4), one then deduces that

$$
\sum_{m \neq n} e^{i \tilde{A}\left(\chi_{m}-\chi_{n}\right)} c_{m}^{*} c_{n} C_{m n}=\sum_{m \neq n} c_{m}^{*} c_{n} C_{m n}
$$

To conclude, we will use the following notion. A group of energy levels of $\mathcal{O}^{(N)}$ is said to be equally spaced if there exists a set of integers $\{p, q, r, s\} \in\{1,2, \ldots, N\}^{4}$ such that

$$
\chi_{p}-\chi_{q}=\chi_{r}-\chi_{s}
$$

We next suppose that the spectrum of $\mathcal{O}^{(N)}$ is not equally spaced (according to the previous definition) and that the coefficients $C_{m n ; m \neq 0} \neq 0$. Using the fact that the lefthand-side of Eq. B5 is a regular function of the parameter $\tilde{A}$ that is constant if and only if all its coefficients $c_{m}^{*} c_{n} C_{m n}$ are zero, we see that the unique solutions of Eq. (B5) are the eigenvectors $\left|\chi^{(n)}\right\rangle$ of $\mathcal{O}^{(N)}$ for which

$$
\left\{\begin{array}{l}
c_{n}=1, \\
c_{m ; m \neq n}=0 .
\end{array}\right.
$$

It is interesting to note that the two previous hypothesis also give practical conditions on $\mathcal{O}^{(N)}$ and $H_{0}$ in order to determine the set $\mathcal{S}$. Indeed, for the orientation process, we notice that the spectrum of $\cos ^{(N)} \theta$ being equally spaced (the spectrum is symmetric), the dimension of $\mathcal{V}$ is not equal to the maximum dimension $N(N-1)$.

Let us now determine the set $\mathcal{F}$ of fixed points for the strategy $S 2$ in the sudden approximation. In this case, we first clarify the definition of $\mathcal{S}$. It can be shown that $\mathcal{S}$ contains the wave functions $|\psi\rangle$ which fulfill

$$
\Im\left[\left\langle\chi^{(N)}\left|e^{i \tilde{A} \mathcal{O}^{(N)}}\right| \psi\right\rangle\left\langle\psi\left|e^{-i \tilde{A} \mathcal{O}^{(N)}} H_{0}\right| \chi^{(N)}\right\rangle\right]=0
$$

for any value of $\tilde{A}$, with $\Im$ corresponding to the imaginary part. Eq. (B8) can be rewritten as

$$
\sum_{m=1}^{N} \Im\left[c_{N} c_{m}^{*} h_{m N} e^{-i A\left(\chi_{m}-\chi_{N}\right)}\right]=0,
$$

the $h_{m n}$ 's being the matrix coefficients of $H_{0}$ in the basis $\left\{\left|\chi^{(n)}\right\rangle\right\}$. If the spectrum of $\mathcal{O}^{(N)}$ is completely non degenerate, one deduces from Eq. (B9) that

$$
\Im\left[c_{N} c_{m}^{*} h_{m N} e^{-i A\left(\chi_{m}-\chi_{N}\right)}\right]=0
$$

for any value of $m(m \neq N)$. Simple algebra shows that

$$
c_{N} c_{m}^{*} h_{m N}=0 .
$$

If we now assume that $h_{m N} \neq 0$, one obtains that $c_{N}=0$ or $c_{m}=0$. The case $c_{N}=0$ corresponds to the minimum of the projection $\left|\left\langle\psi \mid \chi^{(N)}\right\rangle\right|^{2}$, which is not relevant here. Finally, in the other case, we can conclude that the unique element of $\mathcal{S}$ is $\left|\chi^{(N)}\right\rangle$. We also remark that these two conditions are fulfilled for the orientation process. In this example, we have therefore shown that the limit of the sequence is $\left|\chi^{(N)}\right\rangle$. 


\section{APPENDIX C: THE MOLECULAR ALIGNMENT / ORIENTATION CONTROL STRATEGY : THE INFINITE AND THE FINITE DIMENSIONAL CASES}

In this appendix, we shall focus on the convergence of the sequence in an infinite and a finite dimensional Hilbert space, as only the finite case has been treated in Appendices $\mathrm{A}$ and B We first consider the infinite dimensional case. We will explicitly derive Eqs. 35) and (36). For doing so, we recall the following fundamental commutation relations [4], which will be used below:

$$
\begin{array}{rlr}
{\left[J^{2}, \cos \theta\right]} & = & 2\left(\sigma_{\theta}+\cos \theta\right), \\
{\left[\sigma_{\theta}, \cos \theta\right]} & = & \cos ^{2} \theta-\mathbf{1}, \\
{\left[J^{2}, \cos ^{2} \theta\right]} & = & 4 \cos \theta \sigma+6 \cos ^{2} \theta-2, \\
{\left[\sigma, \cos ^{2} \theta\right]} & = & 2 \cos ^{3} \theta-2 \cos \theta \\
{\left[\cos \theta \sigma_{\theta}, \cos ^{2} \theta\right]} & = & 2 \cos ^{4} \theta-2 \cos ^{2} \theta
\end{array}
$$

where $\sigma_{\theta}=\sin \theta \frac{\partial}{\partial \theta}$. We notice that such identities are not fulfilled in a finite dimensional subspace. We consider the strategy $S 1$ for the alignment and the orientation processes. We assume that the average $\left\langle\cos ^{m} \theta\right\rangle$ reaches a maximum at a time $s_{i}$,

$$
\left.\frac{d}{d s}\left\langle\cos ^{m} \theta\right\rangle\right|_{s_{i}-0}=i\left\langle\left[\varepsilon J^{2}, \cos ^{m} \theta\right]\right\rangle=0,
$$

where $m=1$ for the orientation and $m=2$ for the alignment. A sudden pulse being applied at the time $s_{i}$, we have to calculate the slope

$$
\left.\frac{d}{d s}\left\langle\cos ^{m} \theta\right\rangle\right|_{s_{i}+0}=i\left\langle e^{-i A \cos ^{m} \theta} \varepsilon\left[J^{2}, \cos ^{m} \theta\right] e^{i A \cos ^{m} \theta}\right\rangle .
$$

Using Eqs. (C1) and (C3), one readily obtains for the orientation

$$
\left.\frac{d}{d s}\langle\cos \theta\rangle\right|_{s_{i}+0}=i\left\langle\varepsilon\left[J^{2}, \cos \theta\right]\right\rangle+2 \varepsilon A\left(\mathbf{1}-\left\langle\cos ^{2} \theta\right\rangle\right) .
$$

For the alignment process, we have

$$
\left.\frac{d}{d s}\left\langle\cos ^{2} \theta\right\rangle\right|_{s_{i}+0}=i\left\langle\varepsilon\left[J^{2}, \cos ^{2} \theta\right]\right\rangle-4 A \varepsilon\left\langle\left[\cos \theta \sigma_{\theta}, \cos ^{2} \theta\right]\right\rangle,
$$

which can be rewritten as

$$
\left.\frac{d}{d s}\left\langle\cos ^{2} \theta\right\rangle\right|_{s_{i}+0}=i\left\langle\varepsilon\left[J^{2}, \cos ^{2} \theta\right]\right\rangle+2 A \varepsilon\left\langle\sin ^{2} 2 \theta\right\rangle .
$$

From Eq. C22 and from the fact that $\left\langle\cos ^{2} \theta\right\rangle<1$ and $\left\langle\sin ^{2} 2 \theta\right\rangle<1$, one deduces that, in both cases, the slope undergoes a change from zero to a finite non-zero value when a pulse is applied, for any amplitude $A$ and any maximum of the average of $\cos ^{m} \theta$. Note that the sign of the slope and the position of the next local maximum depend on the sign of $A$.
We address now the case of finite dimensionality. In such a space, the commutation relations of Eq. (C1) cannot be used and we have to introduce the following standard basis of $s u(N)$ 12] (related here to the eigenstates of $H_{0}$ ),

$$
\left\{\begin{array}{l}
x_{m n}=e_{m n}-e_{n m}, \\
y_{m n}=i\left(e_{m n}+e_{n m}\right), \\
h_{n}=i\left(e_{n n}-e_{n+1, n+1}\right),
\end{array}\right.
$$

where $e_{m n}=|m\rangle\langle n|, 1 \leq n \leq N-1$ and $n<m \leq N$. $|n\rangle$ is the eigenvector of $H_{0}$ with eigenvalue $E_{n}$ [Eq. (2)]. $H_{0}$ and the interaction term $H_{I}$ can be rewritten with respect to this basis as

$$
\left\{\begin{array}{l}
H_{0}=\sum_{n=1}^{N} E_{n} e_{n n}, \\
H_{I}=\sum_{n=1}^{N-1} d_{n}\left(e_{n, n+1}+e_{n+1, n}\right)
\end{array}\right.
$$

where

$$
E_{n+1}=n(n+1)
$$

and

$$
d_{n+1}=\frac{n+1}{\sqrt{(2 n+1)(2 n+3)}} .
$$

Assuming that $\left\langle\left[H_{0}, H_{I}\right]\right\rangle=0$, we now calculate

$$
\frac{d}{d t}\left\langle H_{I}\right\rangle=\left\langle e^{-i A H_{I}}\left[H_{0}, H_{I}\right] e^{i A H_{I}}\right\rangle,
$$

i.e., the derivative of the function $\left\langle H_{I}\right\rangle(t)$ after a sudden pulse.

A straightforward calculation leads to the identities

$$
\left[H_{0}, H_{I}\right]=-\sum_{n=1}^{N-1} \mu_{n} d_{n} x_{n, n+1},
$$

where $\mu_{n}=E_{n+1}-E_{n}$, and

$$
\begin{aligned}
\frac{1}{2}\left[\left[H_{0}, H_{I}\right], H_{I}\right]= & H_{I}^{2} \\
& +\sum_{n=1}^{N-1}(2 n+1) d_{n}^{2} e_{n+1, n+1} \\
& -\sum_{n=1}^{N}(2 n+3) d_{n}^{2} e_{n n} .
\end{aligned}
$$

From Eq. [C10, one can rewrite Eq. [C13) as

$$
\frac{1}{2}\left[\left[H_{0}, H_{I}\right], H_{I}\right]=H_{I}^{2}-\mathbf{1}+\frac{N^{2}+2 N+1}{2 N+1} e_{N N} .
$$

After expansion according to Campbell-Hausdorf's formula, one gets for the derivative of Eq. [C11) :

$$
\begin{aligned}
\frac{d}{d t}\left\langle H_{I}\right\rangle= & 2 A\left(1-\left\langle V^{2}\right\rangle-\frac{N^{2}+2 N+1}{2 N+1}\left|a_{n}\right|^{2}\right) \\
& -i \frac{\left(N^{2}+2 N+1\right) N}{(2 N+1)^{3 / 2} \sqrt{2 N-1}} A^{2}\left(a_{N}^{*} a_{N-1}-a_{N-1}^{*} a_{N}\right) \\
& +O\left(A^{3}\right),
\end{aligned}
$$


where the $a_{n}$ 's are the coefficients of the wave function in the basis of $|n\rangle$ 's. We notice that Eq. (C15) is very similar to Eq. C4 except for a few terms. If $A$ is small enough, the evolution of the average $\left\langle H_{I}\right\rangle$ will depend on the boundary population $\left|a_{n}\right|^{2}$.

\section{APPENDIX D: ANALYTICAL ESTIMATIONS OF DYNAMICAL PARAMETERS}

In Sec. IV the dynamics were investigated through numerical tests. In this appendix, we give a few analytical estimations of dynamical parameters such as the time between pulses. Nevertheless, it is noted that, owing to the complexity of complete analytical calculations, only rough estimations can be obtained.

We consider the orientation dynamics and the strategy $S 1$. The time $\Delta t$ between two pulses is assumed to be small enough, which means that only the last kicks of the sequence will be correctly described (This point is clearly illustrated in Fig. 4). We now analyze the evolution of $\langle\cos \theta\rangle(s)$ between the times $s_{i}$ (taken as 0 for simplicity) and $s_{f}$, a sudden pulse being applied at $s_{i}$. We have

$$
\langle\cos \theta\rangle(s)=\left\langle e^{-i A \cos \theta} e^{i \varepsilon J^{2} s} \cos \theta e^{-i \varepsilon J^{2} s} e^{i A \cos \theta}\right\rangle,
$$

where $s=t / \tau$ is the rescaled time. Expanding the two time exponentials and neglecting terms of order greater than 2 in $s,\langle\cos \theta\rangle$ can be rewritten in the form

$$
\begin{aligned}
\langle\cos \theta\rangle(s)= & \langle\cos \theta\rangle(0) \\
& +i \varepsilon s\left\langle e^{-i A \cos \theta}\left[J^{2}, \cos \theta\right] e^{i A \cos \theta}\right\rangle \\
& -\frac{1}{2} \varepsilon^{2} s^{2}\left\langle e^{-i A \cos \theta}\left[J^{2},\left[J^{2}, \cos \theta\right]\right] e^{i A \cos \theta}\right\rangle .
\end{aligned}
$$

As $\left\langle\left[J^{2}, \cos \theta\right]\right\rangle(0)=0$, the proof of Appendix [B] shows that

$$
i \varepsilon s\left\langle e^{-i A \cos \theta}\left[J^{2}, \cos \theta\right] e^{i A \cos \theta}\right\rangle=2 \varepsilon A s\left(1-\left\langle\cos ^{2} \theta\right\rangle\right) .
$$

We next simplify the third term of Eq. (D2). Using the commutation relations 44]

$$
\left\{\begin{array}{l}
{\left[J^{2}, \sigma_{\theta}\right]=2 \cos \theta J^{2},} \\
{\left[J^{2}, \cos \theta\right]=\sigma_{\theta}+\cos \theta}
\end{array}\right.
$$

where $\sigma_{\theta}=\sin \theta \frac{\partial}{\partial \theta}$, one arrives at

$$
\begin{aligned}
\left\langle e^{-i A \cos \theta}\left[J^{2},\left[J^{2}, \cos \theta\right]\right] e^{i A \cos \theta}\right\rangle & =4\left\langle\cos \theta J^{2}\right\rangle \\
& +8 i A\left\langle\sigma_{\theta} \cos \theta\right\rangle \\
& +4 A^{3}\left\langle\cos \theta-\cos ^{3} \theta\right\rangle \\
& +4 i A\left(1+\left\langle\cos ^{2} \theta\right\rangle\right) .
\end{aligned}
$$

Introducing the operators

$$
\left\{\begin{array}{l}
\Re\left[\left\langle\sigma_{\theta} \cos \theta\right\rangle\right]=-\frac{1}{2}\left(1+\left\langle\cos ^{2} \theta\right\rangle\right), \\
\Im\left[\left\langle\sigma_{\theta} \cos \theta\right\rangle\right]=\frac{1}{2 i}\left\langle\sigma_{\theta} \cos \theta+\cos \theta \sigma_{\theta}+2 \cos ^{2} \theta\right\rangle,
\end{array}\right.
$$

where $\Re$ and $\Im$ correspond to the real and the imaginary part of a complex number, respectively, Eq. (D2) becomes

$$
\begin{aligned}
\langle\cos \theta\rangle(s) & =\langle\cos \theta\rangle(0)+2 \varepsilon A s\left(1-\left\langle\cos ^{2} \theta\right\rangle\right) \\
& -\frac{1}{2} \varepsilon^{2} s^{2}\left[4\left\langle\cos \theta J^{2}\right\rangle-8 A \Im\left(\left\langle\sigma_{\theta} \cos \theta\right\rangle\right)\right. \\
& \left.+4 A^{2}\left\langle\cos \theta-\cos ^{3} \theta\right\rangle\right] .
\end{aligned}
$$

Equation (D7) allows us to explicitly determine the time $s_{f}$ :

$$
\begin{aligned}
\Delta s & =s_{f}-s_{i} \\
& =\frac{2 A\left(1-\left\langle\cos ^{2} \theta\right\rangle\right)}{\varepsilon\left[4\left\langle\cos \theta J^{2}\right\rangle-8 A \Im\left(\left\langle\sigma_{\theta} \cos \theta\right\rangle\right)+4 A^{2}\left\langle\cos \theta-\cos ^{3} \theta\right\rangle\right]} .
\end{aligned}
$$

Up to this point, the calculation involves no approximation on the area of the field $A$, as only the time delays between the pulses are assumed to be small. In order to highlight different dynamical behaviors, we now consider the two limits $A \gg 1$ and $A \ll 1$. It can be shown that

$$
\Delta s=\frac{1-\left\langle\cos ^{2} \theta\right\rangle}{2 \varepsilon A\left(\left\langle\cos \theta-\cos ^{3} \theta\right\rangle\right)}=\frac{k_{>}}{A}
$$

if $A \gg 1$ and

$$
\Delta s=\frac{A\left(1-\left\langle\cos ^{2} \theta\right\rangle\right)}{2 \varepsilon\left\langle\cos \theta J^{2}\right\rangle}=k_{<} A
$$

if $A \ll 1 . k_{>}$and $k_{<}$are two constants that do not depend on $A$. They can be estimated by assuming that the wave function is close enough to the optimal state $\left|\chi^{(N)}\right\rangle$. The first limit corresponds to the strategy suggested in Ref. [23], where the area $A$ increases with the number of kicks. From Eq. (D9), we note that the expression of the focusing time [23, 45, 46] is derived via a quantum calculation, this time being obtained in Ref. 46] through classical or semi-classical analyses.

Due to the moderate intensity of the pulses used in our strategy (for instance, $A=1$ in Fig. 4), it can be approximately described by Eq. (D10). We recall that the choice of the strength of the kicks is crucial for remaining in the finite dimensional subspace $\mathcal{H}_{m=0}^{(N)}$. This point is clearly illustrated in Fig. 9, which gives a view of the orientation dynamics under the effect of a train of 30 HCPs of area $A=1$. For the last ten pulses, the arithmetic average of the time delays between successive kicks is of the order of $\Delta t / T_{\text {rot }} \simeq 5.6 \times 10^{-3}$. This result can also be derived from the analytical formula of Eq. (D10). Using the eigenstate $\left|\chi^{(5)}\right\rangle$, we obtain $k_{<} \simeq 5 \cdot 10^{-3}$ and

$$
\frac{\Delta t}{T_{\text {rot }}}=\frac{\varepsilon}{\pi} \Delta s=k_{<} A=5.10^{-3},
$$

as $A=1$. Finally, it is noted that we can also estimate the difference $\Delta\langle\cos \theta\rangle=\langle\cos \theta\rangle\left(s_{f}\right)-\langle\cos \theta\rangle\left(s_{i}\right)$ 


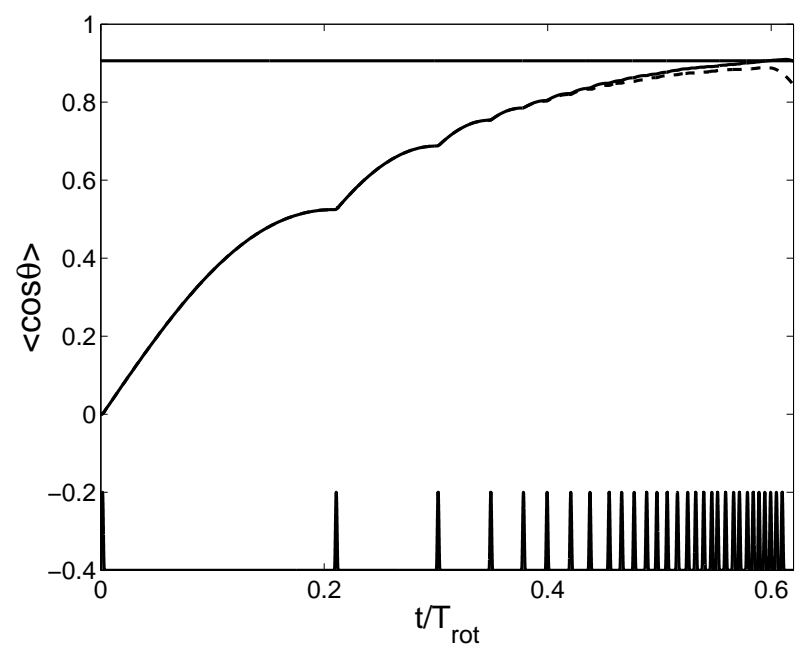

FIG. 9: Orientation dynamics during a train of 30 HCPs. $\varepsilon$ is taken to be 0.01 . between two successive maxima. Straightforward calculation leads to

$$
\Delta\langle\cos \theta\rangle=\frac{\left(1-\left\langle\cos ^{2} \theta\right\rangle\right)^{2} A^{2}}{2\left\langle\cos \theta J^{2}\right\rangle},
$$

in the hypothesis that $A \ll 1$.
[1] W. Warrer, H. Rabitz and M. Dahleh, Science 259, 1581 (1993).

[2] H. Stapelfeldt and T. Seideman, Rev. Mod. Phys. 75, 543 (2003).

[3] O. Atabek and C. M. Dion and A. Ben Haj Yedder, J. Phys. B 36, 4667 (2003).

[4] M. A. Nielsen and I. L. Chuang, Quantum computation and quantum information" (Cambridge Univ. Press, Cambridge, U. K., 2000).

[5] J. I. Cirac and P. Zoller, Phys. Rev. Lett. 74, 4091 (1995).

[6] K. M. Fonseca Romera, G. Useche Laverde and F. Torres Ardila, J. Phys. A 36, 841 (2003).

[7] C. M. Dion, A. Ben Haj Yedder, E. Cancès, A. Keller, C. L. Bris and O. Atabek, Phys. Rev. A 65, 063408 (2002).

[8] H. Rabitz, R. de Vivie-Riedle, M. Motzkus and K. Kompa, Science 288, 824 (2000).

[9] M. D. Girardeau, M. Ina, S. Schirmer and T. Gulsrud, Phys. Rev. A 55, 3 (1997).

[10] M. D. Girardeau, S. G. Schirmer, J. V. Leahy and R. M. Koch, Phys. Rev. A 58, 2684 (1998).

[11] V. Ramakrishna, M. V. Salapaka, M. Dahleb, H. Rabitz and A. Peirce, Phys. Rev. A 51, 960 (1995).

[12] H. Fu, S. G. Schirmer and A. I. Solomon, J. Phys. A 34, 1679 (2001).

[13] D. Sugny, A. Keller, O. Atabek, D. Daems, C. M. Dion, S. Guérin and H. R. Jauslin, Phys. Rev. A 69, 033402 (2004).

[14] N. V. Vitanov, T. Halfmann, B. W. Shore and K. Bergmann, Ann. Rev. Phys. Chem. 52, 763 (2001).

[15] S. Guérin and H. R. Jauslin, Adv. Chem. Phys. 125, 147 (2003).

[16] S. G. Schirmer, A. D. Greentree, V. Ramakrishna and H. Rabitz, J. Phys. A 35, 8315 (2002).

[17] W. Zhu and H. Rabitz, J. Chem. Phys, 110, 7142 (1999).

[18] P. R. Brooks, Science 193, 11 (1976).

[19] T. Seideman, Phys. Rev. A 56, R17 (1997).

[20] T. Seideman, J. Chem. Phys. 111, 4397 (1999).

[21] F. J. Aoiz, Chem. Phys. Lett. 289, 132 (1998).

[22] S. Lloyd and S. L. Braunstein, Phys. Rev. Lett. 82, 1784
(1999).

[23] I. S. Averbukh and R. Arvieu, Phys. Rev. Lett. 87, 163601 (2001).

[24] M. J. J. Vrakking and S. Solte, Chem. Phys. Lett. 271, 209 (1997).

[25] S. Guérin , L. P. Yatsenko, H. R. Jauslin, O. Faucher and B. Lavorel, Phys. Rev. Lett. 88, 233601 (2002).

[26] B. Friedrich and D. Herschbach, Phys. Rev. Lett. 74, 4623 (1995).

[27] N. Sangouard, S. Guérin, M. Amniat-Talab and H. R. Jauslin, Phys. Rev. Lett. 93, 223602 (2004).

[28] C. M. Dion, A. Keller and O. Atabek, Eur. Phys. J. D 14, 249 (2001).

[29] N. E. Henriksen, Chem. Phys. Lett. 312, 196 (1999).

[30] A. Keller, C. M. Dion and O. Atabek, Phys. Rev. A 61, 023409 (2000).

[31] C. M. Dion, A. D. Bandrauk, O. Atabek, A. Keller, H. Umeda and Y. Fujimura, Chem. Phys. Lett. 302, 215 (1999).

[32] M. Machholm and N. E. Henriksen, Phys. Rev. Lett. 87, 193001 (2001).

[33] D. Sugny, A. Keller, O. Atabek, D. Daems, S. Guérin and H. R. Jauslin, Phys. Rev. A 69, 043407 (2004).

[34] D. Daems, A. Keller, S. Guérin, H. R. Jauslin and O. Atabek, Phys. Rev. A 67, 052505 (2003).

[35] M. Machholm, J. Chem. Phys. 115, 10724 (2001).

[36] J. Ortigoso, M. Rodriguez, M. Gupta and B. Friedrich, J. Chem. Phys. 110, 3870 (1999).

[37] A. Ben Haj Yedder, A. Auger, C. M. Dion, E. Cancès, A. Keller, C. Le Bris and O. Atabek, Phys. Rev. A 66, 063401 (2002).

[38] Shlomo E. Sklarz, D. J. Tannor and N. Khaneja, Phys. Rev. A 69, 053408 (2004).

[39] D. J. Tannor and A. Bartana, J. Phys. Chem. A 103, 10359 (1999).

[40] A. Beige, D. Braun, B. Tregenna and P. L. Knight, Phys. Rev. Lett. 85, 1762 (2000).

[41] D. A. Lidar, D. Bacon, J. Kempe and K. B. Whaley, Phys. Rev. A 63, 022307 (2001). 
[42] E. Merzbacher, Quantum Mechanics (Wiley, New York, 1970).

[43] S. G. Schirmer, H. fu and A. I. Solomon, Phys. Rev. A 63, 063410 (2001).

[44] M. Joyeux and D. Sugny, Can. J. Phys. 80, 1459 (2002).
[45] M. Leibscher, I. Sh. Averbukh and H. Rabitz, Phys. Rev. Lett. 90, 213001 (2003).

[46] M. Leibscher, I. Sh. Averbukh, P. Rozmej and R. Arvieu, Phys. Rev. A 69, 032102 (2004). 